\title{
COEXISTENCE THEOREMS OF STEADY STATES FOR PREDATOR-PREY INTERACTING SYSTEMS
}

\author{
LIGE LI
}

\begin{abstract}
In this paper we give necessary and sufficient conditions for the existence of positive solutions of steady states for predator-prey systems under Dirichlet boundary conditions on $\Omega \Subset \mathbf{R}^{n}$. We show that the positive coexistence of predatorprey densities is completely determined by the "marginal density," the unique density of prey or predator while the other one is absent, i.e. the $\left(u_{0}, 0\right)$ or $\left(0, v_{0}\right)$. More specifically, the situation of coexistence is determined by the spectral behavior of certain operators related to these marginal densities and is also completely determined by the stability properties of these marginal densities. The main results are Theorems 1 and 4.2 .
\end{abstract}

1. Main results. In this paper we give necessary and sufficient conditions for the existence of positive solutions to the following system:

$$
\left\{\begin{array}{l}
\Delta u+u M(u, v)=0, \\
d \Delta v+v(g(u)-m(v))=0, \\
\left.(u, v)\right|_{\partial \Omega}=(0,0), \quad \Omega \Subset \mathbf{R}^{n},
\end{array}\right.
$$

where $\Delta$ is the Laplacian $\Delta u=\sum_{i=1}^{n} u_{x_{i} x_{i}} \cdot M$ is a $C^{1}$ function and is globally Lipschitzian, and is assumed to satisfy the so-called prey growth rate conditions (see Figure 1)

$$
\left(\mathrm{M}_{1}\right) M_{v}(u, v)<0 \text { for } u, v \geqslant 0 .
$$

\section{In addition}

$$
\begin{aligned}
& \left(\mathrm{M}_{2}\right) M_{u}(u, 0)<0 \text { for } u \geqslant 0 . \\
& \left(\mathrm{M}_{3}\right) \text { There exists a constant } C_{0}>0 \text { such that } M(u, 0)<0 \text { for } u>C_{0} . \\
& \left(\mathrm{M}_{4}\right) M(0,0)>0 .
\end{aligned}
$$

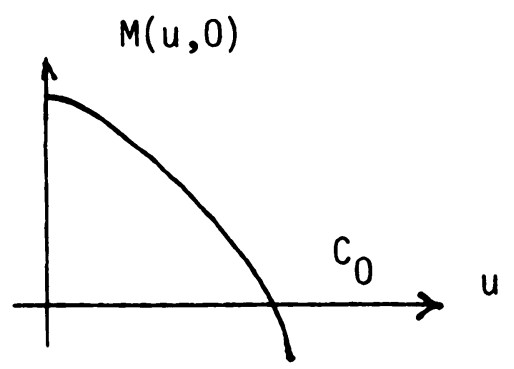

FIGURE 1

Received by the editors September 9, 1986 and, in revised form, January 12, 1987

1980 Mathematics Subject Classification (1985 Revision). Primary 35J60.

$$
\begin{aligned}
& \text { (1) } 1988 \text { American Mathematical Socicty } \\
& \text { (0)(0)2-9947/88 } \$ 1.00+\$ .25 \text { per page }
\end{aligned}
$$


The functions $g$ and $m$ belong to $C^{1}(\mathbf{R})$ and are increasing functions satisfying $g(0)-m(v)<0$ for $v$ larger than some constant $C_{1}$.

In order to state the main result a lemma is in order.

LEMMA 1.1. Let $\phi$ be a strictly decreasing function, $\phi(u) \leqslant 0$ for large $u$.

(i) If $\phi(0)>\lambda_{1}$, then the equation $-\Delta u=u \phi(u),\left.u\right|_{\partial \Omega}=0$ has a unique positive solution.

(ii) If $\phi(0) \leqslant \lambda_{1}$ then the above equation has no positive solution, i.e. $u \equiv 0$ is the only nonnegative solution.

Here $\lambda_{1}$ is the first eigenvalue of $-\Delta$ for the region $\Omega$ with homogeneous Dirichlet condition.

Lemma 1.1 and its proof are well known, see, for example, [4]. Under the above hypotheses about the functions $M, g$ and $m$ the system (1) is a steady state system for the predator-prey problem. We shall see the importance to the system (1) of the component $u$ or $v$ while the other one is absent, that is, the solution $\left(u_{0}, 0\right)$ and $\left(0, v_{0}\right)$ of $(1)$ under certain conditions. According to Lemma 1.1, we have the unique solution $\left(u_{0}, 0\right)$ to $(1)$ with $u_{0}>0$ provided $M(0,0)>\lambda_{1}$, and the unique solution $\left(0, v_{0}\right)$ to (1) with $v_{0}>0$ provided $g(0)>\lambda_{1} d+m(0)$ and $m$ is strictly increasing. Notice that the latter case means a positive density of predator could exist even without the prey, the food source. In and only in this case, in order to get a positive prey density coupled to a predator density, we make an additional hypothesis:

(MM) $M(0, v) \geqslant M(u, v)$ for $u, v \geqslant 0$.

Biologically, (MM) indicates that a maximal birthrate of prey occurs at its absent state.

Our first result is the following theorem.

THEOREM 1.A. If $m \not \equiv 0$, then the positive solution $(u, v)$ of (1) has a priori bounds $0 \leqslant u \leqslant B_{1}, 0 \leqslant u \leqslant B_{2}$.

(A.1) If $M(0,0) \leqslant \lambda_{1}, g(0) \leqslant \lambda_{1} d+m(0)$, then $(0,0)$ is the only nonnegative solution of (1).

(A.2) If $g(0)<\lambda_{1} d+m(0)$, then (1) has a positive solution $(u, v)$ iff $M(0,0)>\lambda_{1}$ and the first eigenvalue of the operator $\mathscr{A}=d \Delta+\left[g\left(u_{0}\right)-m(0)\right] I$ is positive.

(A.3) If $g(0)>\lambda_{1} d+m(0)$, we assume (MM). Then (i) has a positive solution $(u, v)$ iff $M(0,0)>\lambda_{1}$ and the first eigenvalue of the operators $\mathscr{A}=d \Delta+$ $\left[g\left(u_{0}\right)-m(0)\right] I$ and $\mathscr{B}=\Delta+M\left(0, v_{0}\right) I$ are both positive.

THEOREM 1.B. In case $m \equiv 0$, we assume in addition $g(0)<0$ and $\left(\mathrm{M}_{5}\right)$ : There exists $\theta>0$ such that $M_{v}(u, v)<-\theta$ for $0 \leqslant u \leqslant C_{0}, v>0$. Then there are a priori bounds $B_{1}, B_{2}$ for a positive solution of (1). Moreover,

(B.1) If $M(0,0) \leqslant \lambda_{1}$, then $(0,0)$ is the only nonnegative solution.

(B.2) (1) has a positive solution $(u, v)$ iff $M(0,0)>\lambda_{1}$ and the first eigenvalue of $\mathscr{A}=d \Delta+g\left(u_{0}\right) I$ is positive.

REMARK. (1) From the biological point of view the case of (A.2) rules out the possibility that the predator has positive density in the absence of prey. 
(2) Theorem 1 includes many well-known systems and results as special cases. Examples include the quadratic system

$$
\begin{aligned}
& M(u, v)=-a u-b v+c, \\
& g(u)-m(v)=e u-f v \pm g, \quad m(v)=f v, g(u)=c u \pm g,
\end{aligned}
$$

where $a, b, c, e, f, g$ are positive numbers; see $[5,8,12,14,17]$. It is interesting to mention here that the usual monotone technique does not work very well for the above system because the existence of a small positive lower solution will require somewhat severe restrictions on the above system. As a consequence many models are ruled out, for example, when $f=0$ (see below).

$$
\begin{aligned}
& M(u, v)=-a u-b v+c, \\
& g(u)=u-\gamma, \quad m \equiv 0, a, b, c, \gamma>0 .
\end{aligned}
$$

This case was only discussed as an ODE system before. In the PDE case one has to estimate the a priori bound of the solution $v$. Our Theorem 1.B solves this problem.

$$
\begin{aligned}
& M(u, v)=\phi(u)-v \psi(u), \\
& g(u)=k u \psi(u)-\gamma, \quad m \equiv 0,
\end{aligned}
$$

where $\psi(u)=c /(d+u), \phi$ satisfies

$\left(\Phi_{1}\right) \phi(0)>0, \phi^{\prime}(u)<0$ for $u>0$,

$\left(\Phi_{2}\right) \phi(u)<0$ for some constant $u>C_{0}>0$.

2. Preliminary theorems and lemmas. In this section we introduce two theorems due to E. N. Dancer and N. Alikakos and give improvements of them. Additionally, we shall prove some lemmas which are useful in the sequel.

(A) We first follow the notations given in Dancer [11]. Let $E$ be a Banach space. $W=E$ is called a wedge in $E$ if (i) $W$ is a closed convex set and (ii) $\alpha W \subseteq W$ for every real $\alpha \geqslant 0$. A wedge $W$ is called a cone if $W \cap(-W)=\{0\}$. Let $W_{y}=\{x \in$ $E \mid y+\gamma x \in W$ for some $\gamma>0\}$ where $y$ is an arbitrary element in the wedge $W$. Then:

(i) $W_{y}=\{x \in E \mid y+\theta x \in W$ for $0 \leqslant \theta \leqslant \gamma$, for some $\gamma>0\}$.

(ii) $W_{y}$ is convex, $W_{y} \supseteq\{-y\} \cup\{y\} \cup W$.

(iii) $W_{y}$ is also a wedge.

Let $S_{y}=\left\{x \in \bar{W}_{y} \mid-x \in \bar{W}_{y}\right\}$; then $S_{y}$ is a linear subspace of $E$. Let $L$ be a compact linear operator in $E$ with $L\left(\bar{W}_{y}\right) \subseteq \bar{W}_{y} . L$ is said to have property $\alpha$ if there is a $t \in(0,1)$ and an element $w \in \bar{W}_{y} \backslash S_{y}$ such that $w-t L w \in S_{y}$. Dancer's theorem can be stated as follows (see $\$ \S 1,2$ of [11]).

THEOREM D. Let $W$ be a wedge in a Banach space $E$ and $A: W \rightarrow W$ be a compact map with fixed point $A y_{0}=y_{0} \in W$. Let $L=A^{\prime}\left(y_{0}\right)$ be the Fréchet derivative of $A$ at $y_{0}$. Then $L$ maps $\bar{W}_{y_{0}}$ into itself.

(i) Assume $I-L$ is invertible on $E$ and $L$ has property $\alpha$ on $\bar{W} y_{0}$, then $\operatorname{index}_{W}\left(A, y_{0}\right)=0$.

(ii) Assume also that $I-L$ is invertible on $E$. If $L$ does not have property $\alpha$, then $\operatorname{index}_{W}\left(A, y_{0}\right)= \pm 1$. 
Note. By index ${ }_{W}(A, y)$ we mean the degree $\operatorname{deg}_{W}(I-A, U(y), 0)$, where $U(y)$ is a small neighborhood of $y$ in $W$. It is well known that $\operatorname{deg}_{W}(I-A, U(y), 0)$ does not depend on the choice of $U(y)$ as long as it is small enough. Notice that in order to define a Leray-Schauder degree, $W$ should be a retract in the Banach space $E$. By an important theorem of Dugundji, every nonempty closed convex subset of a Banach space $E$ is a retract of $E$. Our wedge is hence a retract of $E$. $\operatorname{deg}_{W}(I-A, U, 0) \neq 0$ implies that there is a fixed point $y \in U$ of $A$. For more details see [2].

In investigating the quadratic system

$$
-\Delta u=a u(1-u)-u v, \quad-d \Delta v=v(-v+m(u-\gamma)),\left.\quad(u, v)\right|_{\partial \Omega}=(0,0),
$$

where $a>\lambda_{1}, m$ and $\gamma$ are positive constants with $0<\gamma<1$, Dancer [12] in 1984 gave a necessary and sufficient condition for the existence of a positive solution to the above system. His proof was based on Theorem D, but this theorem cannot be applied to the above system, since the condition that $I-L$ is invertible on $E$ does not hold. In order to apply the index theorem to our elliptic system (1), we give an improvement to Theorem D as follows.

TheOREM $\mathrm{D}^{\prime}$. Let $y_{0} \in E$ be a fixed point of $A$.

(i) Assume $I-L$ is invertible on $\bar{W}_{y_{0}} \backslash\{0\}$. If $I-L: \bar{W}_{y_{0}} \rightarrow \bar{W}_{y_{0}}$ is not a surjective map, then $\operatorname{index}_{W}(A, y)=0$.

(ii) Assume $I-L$ is invertible on $\bar{W}_{y_{i}}$. If $L$ does not have property $\alpha$ then $\operatorname{index}_{W}(A, y)= \pm 1$.

Theorem $\mathrm{D}^{\prime}$ can be proved by a straightforward modification of the proof of Theorem $\mathrm{D}$ in [11].

(B) Next we state a result obtained by N. Alikakos [1] in 1979. (See $§ 4$ of [1].)

THEOREM A. ${ }^{1}$ Let $(u, v)$ be a positive local (in $\left.t\right)$ solution of

$$
\begin{aligned}
& u_{t}=d_{0} \Delta u+f(u)-\phi(u, v), \\
& v_{t}=d_{1} \Delta v-g(v)+k \phi(u, v), \\
& \left.\left(\frac{\partial u}{\partial n}, \frac{\partial v}{\partial n}\right)\right|_{\partial \Omega}=(0,0),
\end{aligned}
$$

where $0 \leqslant u(x, 0) \leqslant \bar{u}, 0 \leqslant v(x, 0) \leqslant \bar{v}, 0 \leqslant \phi(u, v) \leqslant p(u) v^{\sigma}$, the function $p(u)$ is a continuous monotone nondecreasing function of $u, 1 \leqslant \sigma<(n+2) / n, n=\operatorname{dim} \Omega$, $f(0)=0=f\left(C_{0}\right)$ wher $f(x) \leqslant 0$ for $x \geqslant C_{0}$, and $g(v) \geqslant \mu v$ for some $\mu>0$ and all $v \geqslant 0$. Then $u, v$ can be extended to a global solution over $0 \leqslant t<\infty$ and

$$
\varlimsup_{t \rightarrow x} \max _{x \in \Omega} v(x, t) \leqslant C
$$

where the constant $C$ is independent of the upper bounds $\bar{u}, \bar{v}$ of the initial data.

\footnotetext{
'In reviewing this paper, the referee pointed out that in Theorem $A$ and Theorem $A$ ' below, it is necessary to emphasize the strictness of the second inequality of $1 \leqslant \sigma<(n+2) / n$.
} 
The proof of Theorem A in [1] is based on a result of Conway and Smoller (see Theorem 3.2 of [9]) which says that $\varlimsup_{t \rightarrow \infty}\|v(x, t)\|_{L^{1}}<C_{1}, C_{1}$ being some positive constant.

A modification and improvement of Theorem A can be obtained as follows.

THEOREM A'. (i) If in Theorem A the boundary condition $\left.(\partial u / \partial n, \partial v / \partial n)\right|_{\partial \Omega}=$ $(0,0)$ is replaced by $\left.(u, v)\right|_{\partial \Omega}=(0,0)$ then $\varlimsup_{t \rightarrow \infty} \max V(x, t)<C^{\prime}$, where $C^{\prime}$ does not depend on $\bar{v}$ (but might depend on $\bar{u}$ ) and $C^{\prime}$ does not depend on the diffusion rate $d_{0}$.

(ii) For $\partial v / \partial t=d \Delta v-g(v)+a(x) \phi(v), d>0,\left.v\right|_{\partial \Omega}=0, v \geqslant 0, g(v) \geqslant \mu v$, $\mu>0, \quad 0 \leqslant \phi(v) \leqslant v^{\sigma}$ with $1 \leqslant \sigma<(n+2) / n$, and $a(x)$ is bounded. If $\varlimsup_{t \rightarrow t_{\max }} \int_{\Omega} v(x, t) \leqslant C_{1}$ where $C_{1}$ is independent of the bound of $v(0, x)$, then $\overline{\lim }_{t \rightarrow \infty} \max _{x \in \bar{\Omega}} v(x, t) \leqslant C$, where $C$ is independent of the bound of the initial data $v(0, x)$.

The proof of Theorem $A^{\prime}$ is based on the technique employed in [1] combined with the following facts. A positive solution with homogeneous Dirichlet boundary conditions and certain smoothness of its coefficients must have a nonpositive derivative along the outward normal: $\partial u /\left.\partial n\right|_{a s s} \leqslant 0, \partial v /\left.\partial n\right|_{\partial s} \leqslant 0$. Hence appropriate results in Conway-Smoller [9] still hold under the Dirichlet boundary condition, namely, $\overline{\lim }_{t \rightarrow \infty}\|v(x, t)\|_{I^{\prime}}<C_{1}^{\prime}$. The $C_{1}^{\prime}$ depends on $\operatorname{Max}\left(C_{0}, \bar{u}\right), k$ (or the bound of $a(x)), \mu$, meas $(\Omega)$ and the maximum value of $f$ on $\left[0, \operatorname{Max}\left(C_{0}, \bar{u}\right)\right]$. The equation itself gives the following kinetic energy inequality

$$
\frac{d}{d t}\left(\frac{1}{2} \int_{\Omega} v^{2} d x\right) \leqslant-d \int_{\Omega}|\nabla v|^{2} d x-\mu \int_{\Omega} v^{2}+\operatorname{Max}|a(x)| \int_{\Omega} v^{\sigma+1} d x .
$$

Applying the Nirenberg-Gagliardo inequality to $\int_{\Omega} v^{\sigma+1} d x$, we get

$$
\begin{aligned}
\frac{d}{d t}\left(\int v^{2}\right) & \leqslant(-2 d+\varepsilon) \int_{\Omega}|\nabla v|^{2}-\mu \int_{\Omega} v^{2}+\operatorname{Max}|a(x)| \cdot \frac{1}{\varepsilon}\left(\int_{\Omega} v d x\right) \\
& <-\mu \int_{\Omega} v^{2}+\operatorname{Max}|a(x)| \cdot \frac{1}{\varepsilon}\left(\int v d x\right)=-\mu \int_{\Omega} v^{2}+\tilde{C} .
\end{aligned}
$$

So all arguments in the proof to Theorem 4.1 in Alikakos [1] will work here for the proof of Theorem A'.

(C) We are now going to give some lemmas which play an important role in the sequel.

Lemma 2.1. Assume $a(x) \in L^{\infty}(\Omega)$. Denote by $\lambda_{1}(L)$ the principal eigenvalue of the linear operator $L$. Let $u$ be a function satisfying $u \geqslant 0$ in $\Omega$ with $\left.u\right|_{a \Omega}=0$.

(i) If $0 \not \equiv(\Delta+a(x)) u \geqslant 0$ then $\lambda_{1}(\Delta+a(x) I)>0$.

(ii) If $0 \not \equiv(\Delta+a(x)) u \leqslant 0$ then $\lambda_{1}(\Delta+a(x) I)<0$.

(iii) If $(\Delta+a(x)) u \not \equiv 0$ then $\lambda_{1}(\Delta+a(x) I)=0$.

Proof. (i) Let $\theta(x)$ be the eigenfunction corresponding to the principal eigenvalue $\mu_{1}=\lambda_{1}(\Delta+a(x) I)$. Without loss of generality, we can assume $\theta(x)>0$ in $\Omega$. Then $0<\int_{\Omega}(\Delta+a(x)) u \theta=\mu_{1} \int_{\Omega} u \theta$, hence $\mu_{1}>0$. (Notice that since $(\Delta+a(x)) u$ $\not \equiv 0$ it follows that $u \neq \equiv$, and $\theta(x)>0$, the "ground state" $\theta$ is known to have nice regularity properties and we have $0<\int_{\Omega}(\Delta+a(x)) u \theta d x$.) 
(ii) We need only to switch the sign in the proof of (i).

(iii) $0=\mu_{1} \int_{\Omega} u \theta d x$ with $u \geqslant 0, u \not \equiv 0, \theta>0$. This implies $\mu_{1}=0$.

Lemma 2.2. If $f \in \operatorname{Lip}(\mathbf{R})$ (i.e. $f$ is Lipschitzian on $\mathbf{R}$ to $\mathbf{R}),-\Delta u \geqslant f(u)$, $-\Delta v \leqslant f(v), u \not \equiv v \leqslant u$ in $\Omega$, then $u>v$ in $\Omega$.

PROOF. It follows immediately from the strong maximum principle that either $u \equiv v$ or $u>v$ in $\Omega$.

LEMma 2.3. Assume $T$ is a compact positive linear operator on an ordered Banach space. Let $u>0$ be a positive element. We have the following conclusions.

(i) If $T u>u$ then $r(T)>1$.

(ii) If $T u<u$ then $r(T)<1$.

(iii) If $T u=u$ then $r(T)=1$. $L$.

Here and throughout this paper, $r(L)$ denotes the spectral radius of the operator

Proof. (iii) $T u=u$ implies that 1 is an eigenvalue with positive eigenfunction $u$. The conclusion now follows from the Krein-Rutman theorem (see Theorem 3.2 in [2]), i.e. $1=r(T)$.

(i) Let $T u>u$ and suppose $r(T) \leqslant 1$. Since $1 \cdot u-T u<0$ and $u>0$, by the Krein-Rutman theorem again, $r(T) \neq 1$. Suppose now $r(T)<1$. Since $a=1$. $(-u)-T(-u)>0$ then by the Fredholm alternative there must be a unique solution $v$ of $a=1 \cdot v-T(v)$ and furthermore, by the Krein-Rutman theorem once again, this solution is positive, therefore $(-u)>0$. This contradiction shows $r(T)>$ 1 .

(ii) $T u<u$ implies $1 \cdot u-T u>0$. A similar argument to that in (i) shows $1>r(T)$.

Lemma 2.4. Let $a(x) \in L^{\infty}(\Omega)$. Then $\lambda_{1}(d \Delta+a I)>0$ implies

$$
r\left[(-\Delta+P)^{-1}\left(d^{-1} a+P\right) I\right]>1
$$

where $P$ is any positive constant satisfying $P>d^{-1}\|a\|_{L^{\infty}}$.

Proof. There is an eigenfunction $\phi_{1}>0$ such that $(d \Delta+a) \phi_{1}=\lambda_{1}(d \Delta+a I) \phi_{1}$ $>0$. Therefore $(-\Delta+P) \phi_{1}<\left(d^{-1} a+P\right) \phi_{1}$, so $\phi_{1}<(-\Delta+P)^{-1}\left(d^{-1} a+P\right) \phi_{1}$. But $(-\Delta+P)^{-1}\left(d^{-1} a+P\right) I$ is a compact positive linear operator, hence by Lemma $2.3, r\left[(-\Delta+P)^{-1}\left(d^{-1} a+P\right)\right]>1$.

(D) In this section we briefly recall some well known facts. If $A$ is a second order uniformly elliptic operator, consider the parabolic equation

$$
u_{t}=A u+f(x, u) \text { on } \Omega \times \mathbf{R}^{+}
$$

and its steady state, the elliptic equation $0=A u+f(x, u)$.

The notion of a solution $u$ of the steady state equation being a stable or asymptotically stable solution of the corresponding parabolic equation can be defined in the usual way, for example, see Chapter 10 in [18]. The following is a version of a well-known result (see Theorem 10.5 in [18]). 
Lemma 2.5. Consider $A u+f(x, u)=0,\left.u\right|_{\partial \Omega}=h(x)$. Let $v_{0}, u_{0}$ be a lower and upper solution to the above equation and let $u$ be the unique solution of it with $v_{0} \leqslant u \leqslant u_{0}$. If $u(x, t)$ is any solution of the parabolic equation $u_{t}=A u+f(x, u)$, $\left.u\right|_{\partial \Omega \times \mathbf{R}_{+}}=h(x)$ with $v_{0} \leqslant u(x, 0) \leqslant u_{0}$, then $u(x, t) \rightarrow u(x)$ as $t \rightarrow \infty$, so $u$ is asymptotically stable.

We need the following lemma.

LEMMA 2.6. If $f(x) / x$ is strictly decreasing for $x>0$ with $f(x)<0$ for $x>$ some constant $C_{0}$, then the positive solution of $-\Delta u=f(u),\left.u\right|_{\partial \Omega}=0$ is asymptotically stable.

Proof. By Lemma 1.1 the positive solution $u_{0}$ to $-\Delta u=f(u)$ with $\left.u\right|_{\partial \Omega}=0$ is unique, and by the general maximum principle it must satisfy $0 \leqslant u_{0} \leqslant C_{0}$. It is easy to see that $u \equiv C_{0}$ is an upper solution while $u \equiv 0$ is a lower solution. (Notice that our assumption about $f(x)$ implies that $f(0)=0$.) Our lemma now follows from Lemma 2.5.

(E) We want to prove the following proposition.

Proposition 2.7. Let $f(u) / u$ be strictly decreasing with $f(u)<0$ for $u>C_{0}$ and let $f^{\prime}(0)>\lambda_{1}$. Thus we have a unique positive solution $u_{0}$ of $-\Delta u=f(u),\left.u\right|_{\text {a } \Omega}=0$. Then $w=0$ is the only solution of the linearization $-\Delta w=f^{\prime}\left(u_{0}\right) w,\left.w\right|_{\text {ว } \Omega}=0$.

Proof. (1) By the general maximum principle $u_{0} \leqslant C_{0}$. Thus by Lemma 2.2 , $0<u_{0}<C_{0}$, so $f\left(u_{0}\right)>0$.

(2) By Lemma 2.6, $u_{0}$ is asymptotically stable with respect to the parabolic equation $u_{t}=\Delta u+f(u),\left.u\right|_{\partial \Omega \times \mathbf{R}}=0$, so the spectrum of the operator $\Delta+f^{\prime}\left(u_{0}\right) \cdot I$ lies on the real semiaxis $(-\infty, 0]$. This proposition claims that $\mu<0$. We shall prove this claim by using bifurcation techniques. Consider now the following equation

$$
-\Delta w=\theta f\left(u_{0}\right) w,\left.\quad w\right|_{\partial \Omega}=0
$$

where $\theta \in[1-\varepsilon, 1+\varepsilon]$ is a positive number close to 1 such that $\theta f^{\prime}(0)>\lambda_{1}$ and therefore the equation

$$
F(u, \theta) \equiv \Delta u+\theta f(u)=0,\left.\quad u\right|_{\partial \Omega}=0
$$

has a unique positive solution $u_{0 \theta}$ for each such given $\theta$ with $f\left(u_{0 \theta}\right)>0$ for the same reason as that in part (1) of this proof.

(3) We are going to show that at $\theta=1$ our problem undergoes some sort of secondary bifurcation. Indeed, what we employ here is the proof of the CrandallRabinowitz bifurcation theorem (see [10 or 18]). Our first step is to verify the usual three conditions under which bifurcation occurs.

(i) First suppose there is a $w_{0} \not \equiv 0$ satisfying $-\Delta w_{0}=f^{\prime}\left(u_{0}\right) w_{0},\left.w_{0}\right|_{a \Omega}=0$; then zero is the principal eigenvalue of the operator $\mathscr{A}=\Delta+f^{\prime}\left(u_{0}\right) I$. By Lemma 2.6, the spectrum of $\mathscr{A}$ lies in $(-\infty, 0]$, so we may assume that $w_{0}(x)>0$ for $x \in \Omega$ and that zero is a simple eigenvalue: $\operatorname{dim}(\operatorname{Ker} \mathscr{A})=1$. Here $\mathscr{A}$ is the Fréchet derivative $D_{u}(\Delta u+\theta f(u))$ at $u_{0}$ and $\theta=1$, where $u_{0}=u_{01}$. 
(ii) Second, by the well-known selfadjoint property of $\Delta+f^{\prime}\left(u_{0}\right) I$ under zero Dirichlet boundary condition and the Fredholm alternative, $\operatorname{codim}(\mathscr{R}(\mathscr{A}))=1$ where $\mathscr{R}(\mathscr{A})$ is the range of $\mathscr{A}$ and $\phi \in \mathscr{R}(\mathscr{A})$ iff $\int_{\Omega} \phi w_{0}=0$.

(iii) We claim $\left.\left[D_{\theta} D_{u}(\Delta u+\theta f(u))\right]\right|_{u=u_{0}, \theta=1}\left(w_{0}\right) \notin \mathscr{R}(\mathscr{A})$. To see this notice that

$$
\left.\left[D_{\theta} D_{u}(\Delta u+\theta f(u))\right]\right|_{u=u_{0}, \theta=1}\left(w_{0}\right)=f^{\prime}\left(u_{0}\right) w_{0},
$$

and

$$
\int_{\Omega} f^{\prime}\left(u_{0}\right) w_{0} w_{0}=-\int_{\Omega}\left(\Delta w_{0}\right) w_{0}=\int_{\Omega}\left|\nabla w_{0}\right|^{2} \neq 0
$$

because $w_{0} \not \equiv 0$ with $\left.w_{0}\right|_{\partial \Omega}=0$. This proves our claim.

Combining (i)-(iii), it follows that at $\theta=1$ we can get some bifurcation about $\Delta u+\theta f(u)=0$ (or equivalently, we can employ the implicit function theorem) and we get the solution

$$
u=u(s)=u_{0}+s\left(w_{0}+\phi(s)\right)
$$

and $1-\varepsilon \leqslant \theta(s) \leqslant 1+\varepsilon$ satisfying $\Delta u+\theta f(u)=0$ with $\phi(0)=0, \theta(0)=1$, where $s$ is in a neighborhood of zero; $\theta(s)$ passes across 1 when $s$ crosses zero and $\phi(s) \in\left(w_{0}\right)^{\perp}=\mathscr{R}(\mathscr{A})$. For small positive $s_{1}$, since $u_{0}, w_{0}>0, \phi(0)=0$, it follows that $u_{1}=u_{0}+s_{1}\left(w_{0}+\phi\left(s_{1}\right)\right)$ is positive in $\Omega$. Notice that

$$
-\Delta u_{0}=f\left(u_{0}\right), \quad-\Delta w_{0}=f^{\prime}\left(u_{0}\right) w_{0} .
$$

Thus we have

$$
\Delta u_{1}=\Delta u_{0}+s_{1} \Delta w_{0}+s_{1} \Delta \phi
$$

or

$$
-\Delta u_{1}=-\Delta u_{0}-s_{1} \Delta w_{0}-s_{1} \Delta \phi=f\left(u_{0}\right)+s_{1} f^{\prime}\left(u_{0}\right) w_{0}-s_{1} \Delta \phi .
$$

On the other hand,

$$
-\Delta u_{1}=\theta\left(s_{1}\right) f\left(u_{1}\right)=\theta\left(s_{1}\right)\left[f\left(u_{0}\right)+f^{\prime}\left(u_{0}\right)\left(s_{1} w_{0}+s_{1} \phi\left(s_{1}\right)\right)+\delta\right]
$$

by using the Fréchet derivative at $u_{0}$ where $\|\delta\| / s_{1} \rightarrow 0$ as $s_{1} \rightarrow 0$. We thus have the following equality:

$$
f\left(u_{0}\right)+s_{1} f^{\prime}\left(u_{0}\right) w_{0}-s_{1} \Delta \phi=\theta\left(s_{1}\right)\left[f\left(u_{0}\right)+f^{\prime}\left(u_{0}\right)\left(s_{1} w_{0}+s_{1} \phi\left(s_{1}\right)\right)+\delta\right] .
$$

A simple manipulation yields

$$
\left(1-\theta\left(s_{1}\right)\right) f\left(u_{0}\right)+s_{1}\left(1-\theta\left(s_{1}\right)\right) f^{\prime}\left(u_{0}\right) w_{0}-s_{1} \Delta \phi=s_{1} f^{\prime}\left(u_{0}\right) \phi\left(s_{1}\right)+\theta\left(s_{1}\right) \delta .
$$

Dividing by $s_{1}$ and multiplying by $w_{0}$, we get

$$
\begin{gathered}
s_{1}^{-1}\left(1-\theta\left(s_{1}\right)\right) f\left(u_{0}\right) w_{0}+\left(1-\theta\left(s_{1}\right)\right) f^{\prime}\left(u_{0}\right) w_{0}^{2} \\
=\left(f^{\prime}\left(u_{0}\right) \phi+\Delta \phi\right) w_{0}+s_{1}^{-1} \delta \theta\left(s_{1}\right) w_{0} .
\end{gathered}
$$

Next take the integral on both sides and notice that

$$
\int_{\Omega}\left(f^{\prime}\left(u_{0}\right) \phi+\Delta \phi\right) w_{0}=\int_{\Omega}\left(f^{\prime}\left(u_{0}\right) w_{0}+\Delta w_{0}\right) \phi=0 .
$$


Letting $s_{1} \rightarrow 0$ we thus have $\theta^{\prime}(0) \int_{\Omega} f\left(u_{0}\right) w_{0}=0$. The statement that $\theta(s)$ passes across 1 at $s=0$ means $\theta^{\prime}(0) \neq 0$, therefore $\int_{\Omega} f\left(u_{0}\right) w_{0}=0$. But $w_{0}>0, f\left(u_{0}\right)>0$. This contradiction proves that $w_{0} \equiv 0$, and hence completes the proof of our proposition.

Note. Smoller, Tromba and Wasserman in [19] obtained interesting results of the one dimensional space setting by employing a one dimensional covering space technique.

\section{A priori bounds.}

(A) $m \not \equiv 0$. The first equation of the system

$$
\left\{\begin{array}{l}
-\Delta u=u M(u, v), \\
-d \Delta v=v[g(u)-m(v)],\left.\quad(u, v)\right|_{\partial \Omega}=(0,0),
\end{array}\right.
$$

can be written as

$$
-\Delta u=u M(u, 0)-u[M(u, 0)-M(u, v)] .
$$

Notice that $M(u, 0)-M(u, v) \geqslant 0$ and $g(u)-g(0) \geqslant 0$ for $u \geqslant 0, v \geqslant 0$. By Lemma 1.1, the problem

$$
-\Delta u=u M(u, 0),\left.\quad u\right|_{\partial \Omega}=0
$$

has a unique positive solution $u_{0}$ provided $M(0,0)>\lambda_{1}$. Since

$$
u M(u, 0)-u[M(u, 0)-M(u, v)] \leqslant u M(u, 0) \text { for } u, v \geqslant 0,
$$

if $(u, v)$ is a positive solution to the system (1), then $u$ is a lower solution to (3.1). By uniqueness it follows immediately that $u \leqslant u_{0}$. Since $M(u, 0)<0$ for $u>C_{0}$, the general maximum principle gives $\sup _{x \in \Omega} u_{0}(x) \leqslant C_{0}$. Therefore $0 \leqslant u \leqslant u_{0}<C_{0}+$ $\varepsilon=B_{1}$.

Now consider $-d \Delta v=v[g(u)-m(v)],\left.v\right|_{\partial \Omega}=0$. The general maximum principle together with the monotonicity of $g$ yields $\sup _{x \in \Omega} m(v(x)) \leqslant g\left(B_{1}\right)$. The upper bound to $v$ then follows from the monotonicity of $m$.

(B) $m \equiv 0$.

In this case consider the corresponding parabolic system

(1.B), $u_{t}=\Delta u+u M(u, v), \quad v_{t}=d \Delta v+v g(u),\left.\quad(u, v)\right|_{\partial \Omega \times \mathbf{R}^{+}}=(0,0)$

with $0 \leqslant u(0, x) \leqslant \bar{u}\left(\leqslant C_{0}\right.$, of course), $0 \leqslant v(0, x) \leqslant \bar{v}$, where $\bar{u}, \bar{v}$ are the bounds of the initial data. We shall use the theory of invariant region and $f$-stability (see [18, Chapter 16]). We shall use the following well-known result (see [18, Chapter 14]).

THEOREM 3.1. Let $D$ be a positive diagonal matrix with $\Sigma$ a rectangle and assume the system $\vec{u}_{t}=D \Delta \vec{u}+f(\vec{u})$ is $f$-stable. Then $\Sigma$ is invariant iff $f$ does not point outward at each point of $\partial \Sigma$.

Using operator semigroup theory (see [13]), one can prove that as long as $f \in C^{1}$ is globally Lipschitzian, the above system is $f$-stable.

We return to the system (1.B) $)_{t}$. Denote by $B_{1}=\max \left(\bar{u}, C_{0}\right)>0$. Then by Theorem 3.1 it is easy to check that the rectangle $0 \leqslant u \leqslant B_{1}, 0 \leqslant v<\infty$ is an invariant region of the system (1.B) ${ }_{t}$. By hypothesis $\left(\mathrm{M}_{5}\right)$ for Theorem 1.B, $M_{v}(u, v)<-\theta$ 
for $0 \leqslant u \leqslant C_{0}, v \geqslant 0$. It follows that there is a positive constant $k>0$ such that $k \min _{0 \leqslant u \leqslant C_{0}}\left|M_{v},(u, v)\right| \geqslant \max _{0 \leqslant u \leqslant C_{0}} g^{\prime}(u)$ with $k$ independent of $u, v$. Rewrite $(1 . B)$, as

$$
\begin{aligned}
& u_{t}=\Delta u+u M(u, 0)-u[M(u, 0)-M(u, v)], \\
& v_{t}=d \Delta v+v[g(0)+g(u)-g(0)], \\
& \left.(u, v)\right|_{\partial \Omega \times \mathbf{R}^{+}}=(0,0) .
\end{aligned}
$$

Use the mean value theorem to get

$$
\begin{aligned}
& u_{t}=\Delta u+u M(u, 0)+u v M_{v}\left(u, \theta_{1} v\right), \\
& v_{t}=d \Delta v+v g(0)+u v g^{\prime}\left(\theta_{2} u\right)
\end{aligned}
$$

where $\theta_{1}, \theta_{2} \in[0,1]$. Integrating both equations over $\Omega$ and noticing that $\partial u /\left.\partial n\right|_{\partial \Omega \times \mathbf{R}} \leqslant 0, \partial v /\left.\partial n\right|_{\partial \Omega \times \mathbf{R}} \leqslant 0(u, v$ are positive solutions) gives

$$
\int_{\Omega}\left(k u_{t}+v_{t}\right) \leqslant k \int_{\Omega} u M(u, 0)+\int_{\Omega} v g(0)+\int_{\Omega} v u\left(k M_{t^{\prime}}+g^{\prime}\right) .
$$

Since $M_{r}<0$ and $k\left|M_{t}\right| \geqslant g^{\prime}$, we thus have

$$
\begin{aligned}
\frac{d}{d t} \int_{\Omega}(k u+v) & =\int_{\Omega} k u_{t}+v_{t} \leqslant k \int_{\Omega} u M(u, 0)+\int_{\Omega} v g(0) \\
& =g(0) \int_{\Omega}(k u+v)+k \int_{\Omega}[u M(u, 0)-g(0) u] \\
& \leqslant g(0) \int_{\Omega}(k u+v)+C^{\prime} .
\end{aligned}
$$

$C^{\prime}$ does not depend on initial data $v(0, x)$. Since $u$ is bounded by $C_{0}$ and $g(0)<0$, by the boundedness of $u(t, x)$ and Gronwall's inequality it follows that $\varlimsup_{t \rightarrow t_{\max }} \int_{\Omega} v(t, x) d x<c$ for some constant $c$, which is independent of the bound of the initial data $v(0, x)$. Using part (ii) of Theorem $\mathrm{A}^{\prime}$ in $\$ 2$, we deduce that there is a constant $B_{2}$ independent of $\bar{v}$ such that $\overline{\lim }_{t \rightarrow \infty} \max v(t, x) \leqslant B_{2}$.

Let us return to the elliptic system

$$
0=\Delta u+u M(u, v), \quad 0=d \Delta v+v g(u),\left.\quad(u, v)\right|_{\text {a } \Omega}=(0,0) .
$$

Then $(u, v)$ is also a solution of the parabolic problem (1.B), with the initial data as $u(x), v(x)$ themselves. The independence of the bound $B_{2}$ to the initial data implies $v(x)=\varlimsup_{\lim _{t \rightarrow \infty}} v(t, x) \leqslant B_{2}$. Notice that since $0 \leqslant u \leqslant B_{1}, B_{2}$ does not depend on $u$ either, in fact, if necessary, let $B_{2}^{\prime}=\max \left(B_{2}, B_{1}\right)$ be the new $B_{2}$. This gives a priori bounds to the solution $(u, v)$ of $(1 . \mathrm{B})$.

4. Proof of Theorems 1.A and 1.B. In this section we shall complete the proof of Theorems 1.A and 1.B.

(I) First we prove the necessity parts of both theorems. Denote the nonnegative solution to (1) by $(\tilde{u}, \tilde{v})$. Since $0 \leqslant \tilde{v} \not \equiv 0$, apply Lemma 2.2 to the second equation in (1), noticing that zero is an obvious solution we get $\tilde{v}(x)>0$ in $\Omega$. Similarly, $\tilde{u}(x)>0$ in $\Omega$. Since $M_{v}(u, v)<0$ for $u, v \geqslant 0$ and $\Delta \tilde{u}+\tilde{u} M(\tilde{u}, \tilde{v})=0$, we have $\Delta \tilde{u}+\tilde{u} M(\tilde{u}, 0) \geqslant 0$, so $\tilde{u}$ is a positive lower solution of the equation

$$
\Delta u+u M(u, 0)=0,\left.\quad u\right|_{\partial \Omega}=0 .
$$


Notice that the constant $C_{0}+\varepsilon$ is an upper solution to the above equation and that $\tilde{u} \leqslant B_{1}=C_{0}+\varepsilon$. It follows that there exists a positive solution $u_{0}$ of the above equation with $0<\tilde{u} \leqslant u_{0} \leqslant B_{1}$. Applying again Lemma 2.2, we have $\tilde{u}<u_{0}$ in $\Omega$. Since $u_{0}>0$ by Lemma 1.1 we have $M(0,0)>\lambda_{1}$. Because $u_{0}>\tilde{u}, \tilde{v}>0$, it follows that

$$
-d \Delta \tilde{v}=\tilde{v}(g(\tilde{u})-m(\tilde{v})) \leqslant \tilde{v}\left(g\left(u_{0}\right)-m(0)\right),
$$

therefore $d \Delta \tilde{v}+\left[g\left(u_{0}\right)-m(0)\right] \tilde{v} \geqslant 0$. Then Lemma 2.1 implies that

$$
\lambda_{1}\left[d \Delta+\left(g\left(u_{0}\right)-m(0)\right) I\right]>0 .
$$

(Letting $m \equiv 0$, we also obtain the necessity for Theorem 1.B.)

In case $g(0)>\lambda_{1} d+m(0)$, we have another trivial solution $\left(0, v_{0}\right)$, since $\tilde{v}>0$ and $-d \Delta \tilde{v}=\tilde{v}(g(\tilde{u})-m(\tilde{v})) \geqslant \tilde{v}(g(0)-m(\tilde{v}))$. So $\tilde{v}$ is an upper solution of the equation

$$
-d \Delta v=v(g(0)-m(v)),\left.\quad v\right|_{\partial \Omega}=0 .
$$

Consider $\delta \phi_{1}$ where $\phi_{1}>0$ is the eigenfunction corresponding to $\lambda_{1}$ of

$$
-\Delta \phi_{1}=\lambda_{1} \phi_{1},\left.\quad \phi_{1}\right|_{\partial \Omega}=0
$$

and $\delta$ is so small that $g(0)-m\left(\delta \phi_{1}\right)>\lambda_{1} d>0$ (by the continuity of $m$ at zero and the assumption that $\left.g(0)>\lambda_{1} d+m(0)\right)$. So

$$
-d \Delta\left(\delta \phi_{1}\right)=\lambda_{1} d \delta \phi_{1}<\delta \phi_{1}\left(g(0)-m\left(\delta \phi_{1}\right)\right)
$$

whence $\delta \phi_{1}>0$ is a positive lower solution to the above equation. It is not hard to prove $\tilde{v} \geqslant \delta \phi_{1}$ provided $\delta$ is small enough. (To see this, we rewrite the second equation of (1) as

$$
-d \Delta \tilde{v}+\tilde{v}(m(\tilde{v})-m(0))=\tilde{v}(g(\tilde{u})-m(0))>0 .
$$

The positivity of the right-hand side is due to the assumption $g(0)-m(0)>\lambda_{1} d>0$ and $g(\tilde{u})-m(0) \geqslant g(0)-m(0)$. Notice that $m(\tilde{v})-m(0) \geqslant 0$, and also that $\tilde{v}$ attains its minimum zero only at $\partial \Omega$, so by the Hopf minimum principle, $\partial \tilde{v} /\left.\partial n\right|_{\partial \Omega}$ $<0$ (here we have used the regularity). Now $\partial \phi_{1} /\left.\partial n\right|_{\partial \Omega} \leqslant 0$ (actually, $\partial \phi_{1} /\left.\partial n\right|_{\partial \Omega}$ $<0)$, since $\partial \Omega$ is a closed set, there is a small $\delta_{1}$ such that $\left.\left(\partial \tilde{v} / \partial n-\delta_{1} \partial \phi_{1} / \partial n\right)\right|_{\partial \Omega}$ $<0$. Since $\left.\left(\tilde{v}-\delta_{1} \phi_{1}\right)\right|_{\partial \Omega}=0$ hence $\tilde{v}-\delta_{1} \phi_{1} \geqslant 0$ in a neighborhood $N$ of $\partial \Omega$. We can find a small positive $\delta_{2}$ such that $\tilde{v}-\delta_{2} \phi_{1}>0$ in $\bar{\Omega} \backslash N$. Let $\delta=\operatorname{Min}\left(\delta_{1}, \delta_{2}\right)$. Then we have $\tilde{v}-\delta \phi_{1} \geqslant 0$ in $\bar{\Omega}$.)

By the well-known lower-upper solution technique and the fact that $v_{0}$ is the unique solution of

$$
-d \Delta v=v(g(0)-m(v)),\left.\quad v\right|_{\partial \Omega}=0,
$$

we deduce $\tilde{v} \geqslant v_{0}$, so we have

$$
0=\Delta \tilde{u}+\tilde{u} M(\tilde{u}, \tilde{v}) \leqslant \Delta \tilde{u}+\tilde{u} M\left(\tilde{u}, v_{0}\right) \leqslant \Delta \tilde{u}+\tilde{u} M\left(0, v_{0}\right) .
$$

Since $\tilde{u}>0$, by Lemma 2.1, $\lambda_{1}\left(\Delta+M\left(0, v_{0}\right)\right)>0$. This completes the proof.

REMARK. We have seen that for any positive solution $(u, v)$ of $(1), u \leqslant u_{0}, v \geqslant v_{0}$ always hold. We have, indeed, by Lemma 2.2, $u<u_{0}, v>v_{0}$. ( $v_{0}$ would be zero provided $g(0) \leqslant \lambda_{1} d+m(0)$.) 
(II) To prove the sufficiency of our condition we confine ourselves to Theorem 1.A. A remark will be given later indicating the modification to the arguments necessary for proving Theorem 1.B. The proof we are going through here is a generalization of that in [12].

From the result of $\S 3$ we have a priori bounds $0 \leqslant u \leqslant B_{1}, 0 \leqslant v \leqslant B_{2}$. Consequently there is a positive number $P$ which is large enough that

$$
\max \left\{\max _{x \in \bar{\Omega}}|M(u(x), v(x))|, \max _{x \in \bar{\Omega}} d^{-1}|g(u(x))-m(v(x))|\right\} \leqslant P .
$$

Let

$$
A(u, v)=(-\Delta+P)^{-1}\left[u M(u, v)+P u, d^{-1} v(g(u)-m(v))+P v\right] .
$$

By the strong maximum principle, $(-\Delta+P)^{-1}$ is a compact linear positive map, so $A$ is a direct sum of compact positive maps. It is clear that $(u, v)$ is a solution of (1) iff $(u, v)$ is a fixed point of $A$ (and this claim has nothing to do with the choice of $P$ as long as $P$ is large enough). Consider the equations

$$
\left\{\begin{array}{l}
-\Delta u=\theta u M(u, v), \\
-d \Delta v=\theta v(g(u)-m(v)),\left.\quad(u, v)\right|_{\partial \Omega}=(0,0),
\end{array}\right.
$$

where $\theta$ is playing the role of a parameter running from zero to infinity. Let $D=\left\{(u, v) \in C_{0}(\Omega) \oplus C_{0}(\Omega) \mid u \leqslant B_{1}+1, v \leqslant B_{2}+1\right\}$ where $B_{1}, B_{2}$ are the a priori bounds of the solution $(u, v)$ of (1). Let $K$ be the positive cone of $C_{0}(\Omega)$ :

$$
K=\left\{f \in C_{0}(\Omega) \mid f(x) \geqslant 0 \text { for } x \in \bar{\Omega}, f(x)=0 \text { for } x \in \partial \Omega\right\} .
$$

From $\S 3$ the upper bounds of $u$ and $v$ are independent of the diffusion rates. (Recall that we are dealing with Theorem 1.A.) So we can assume that the positive solution $\left(u_{\theta}, v_{\theta}\right)$ of $\left(1 . \mathrm{A}_{\theta}\right)$ satisifies $v_{\theta} \leqslant B_{2}, u_{\theta} \leqslant B_{1}$ for all $\theta \in(0, \infty)$. Let

$$
A_{\theta}(u, v)=(-\Delta+P)^{-1}\left[\theta u M(u, v)+P u, d^{-1} \theta v[g(u)-m(v)]+P v\right] .
$$

By the definition of $D, A_{\theta}$ has no fixed point on $\partial D$ for all $\theta \in(0, \infty)$. Hence the homotopy invariance of degree theory tells us that the fixed point index $i\left(A_{\theta}\right.$, int $\left.D, K \oplus K\right)$ is independent of $\theta$. Because the case (A.3) in Theorem 1.A is the hardest one, we shall discuss mainly this case.

Let $\theta \in\left(0, \lambda_{1} / M(0,0)\right) \cap\left(0, \lambda_{1} d /(g(0)-m(0))\right)$, where $\lambda_{1}$ is the first eigenvalue of $-\Delta$ over $\Omega$ under zero Dirichlet boundary conditions. Then for such a choice of $\theta$ the system $(1 . \mathrm{A})_{\theta}$ has as its only nonnegative solution $u \equiv 0, v \equiv 0$. To see this, first notice that under this choice of $\theta, u_{\theta 0} \equiv 0$ is the only nonnegative solution to the equation

$$
-\Delta u=\theta u M(u, 0),\left.\quad u\right|_{\partial \Omega}=0 .
$$

Since $0 \leqslant u_{\theta} \leqslant u_{\theta 0}$ always holds, therefore $u_{\theta} \equiv 0$. Consequently, since $\theta \in$ $\left(0, \lambda_{1} d /(g(0)-m(0))\right)$, by Lemma 1.1 , the equation $-d \Delta v_{\theta}=\theta v_{\theta}\left(g(0)-m\left(v_{\theta}\right)\right)$ with $\left.v_{\theta}\right|_{\partial \Omega}=0$ implies $v_{\theta} \equiv 0$. Thus $(0,0)$ is the only fixed point of $A_{\theta}$ in $K \oplus K$, i.e. for the above choice of $\theta$

$$
i\left(A_{\theta}, \text { int } D, K \oplus K\right)=\operatorname{deg}\left(I-A_{\theta}, N(0,0),(0,0)\right),
$$


where $N(0,0)$ is a small neighborhood of $(0,0)$ in $D$. As is well known for a compact positive operator $A$ with fixed point $A y=y$,

$$
\operatorname{deg}(I-A, U(y), 0)=\operatorname{index}(L, 0, C)
$$

where $U(y)$ is a small neighborhood of $y, C$ is the positive cone, $L$ is the Fréchet derivative of $A$ at $y$ which is of course linear, and index $(L, 0, C)=(-1)^{\sigma}$ where $\sigma$ is the sum of the multiplicities of the eigenvalues of $L$ larger than one. So index $=1$ provided $r(L)<1$.

Now

$$
L=A_{\theta}^{\prime}(0,0)=(-\Delta+P)^{-1}\left[\begin{array}{cc}
\theta M(0,0)+P & 0 \\
0 & d^{-1} \theta(g(0)-m(0))+P
\end{array}\right]
$$

Because $\theta \in\left(0, \lambda_{1} / M(0,0)\right) \cap\left(0, \lambda_{1} d /(g(0)-m(0))\right)$, therefore $r(L)<1$, and we have

$$
i\left(A_{\theta}, \text { int } D, K \oplus K\right)=\operatorname{deg}\left(I-A_{\theta}, N(0,0),(0,0)\right)=\operatorname{index}(L, 0, K \oplus K)=1
$$

for all $\theta$ as above. But this holds for all $\theta>0$ by the homotopy invariance property, including $\theta=1$. Since we are particularly dealing with the case (A.3), so we have three trivial nonnegative solutions of $(1):(0,0),\left(u_{0}, 0\right)$ and $\left(0, v_{0}\right)$. If we can show

$$
\begin{aligned}
0 & =i \operatorname{index}(A,(0,0), K \oplus K)=\operatorname{index}\left(A,\left(U_{0}, 0\right), K \oplus K\right) \\
& =i n d e x\left(A,\left(0, V_{0}\right), K \oplus K\right)
\end{aligned}
$$

then there must be at least a positive solution $(u, v)$ in $D$.

Note. In case (A.2) of Theorem 1.A, we have only two trivial nonnegative solutions of $(1):(0,0),\left(u_{0}, 0\right)$, and need only to prove

$$
0=\operatorname{index}(A,(0,0), K \oplus K)=\operatorname{index}\left(A,\left(u_{0}, 0\right), K \oplus K\right) .
$$

(A) We investigate the case of $y=(0,0)$.

In this case $\bar{W}_{y}=\left\{(f, \phi) \in C_{0}(\Omega) \oplus C_{0}(\Omega) \mid(0,0)+\gamma(f, \phi) \in K \oplus K\right.$ for some $\gamma>0\}$. So $\bar{W}_{y}=K \oplus K$. Let $L=A^{\prime}(0,0)$. Then $I-L$ is not invertible in $E=$ $C_{0}(\Omega) \oplus C_{0}(\Omega)$. (This can be verified when $M(0,0)$ is one of the values $\left\{\lambda_{2}, \lambda_{3}, \ldots, \lambda_{n}, \ldots\right\}$.) We thus appeal to Theorem $\mathrm{D}^{\prime}$ in $\S 2$. We need to check that $I-L$ is invertible in $\bar{W}_{y}=K \oplus K$ and it is not a surjective map on $\bar{W}_{y}$. Suppose that

$$
\left(\begin{array}{l}
r \\
k
\end{array}\right)=A^{\prime}(0,0)\left(\begin{array}{l}
r \\
k
\end{array}\right), \quad \text { where }\left(\begin{array}{l}
r \\
k
\end{array}\right) \in \bar{W}_{y} .
$$

This can be written in another form, namely

$$
\begin{aligned}
& (-\Delta+P)^{-1}(P+M(0,0)) r=r, \\
& (-\Delta+P)^{-1}\left(P+d^{-1}(g(0)-m(0))\right) k=k .
\end{aligned}
$$

From the second equation,

$$
-\Delta k=d^{-1}(g(0)-m(0)) k,\left.\quad k\right|_{\partial \Omega}=0 .
$$


But

$$
d^{-1}(g(0)-m(0))>\lambda_{1},
$$

whence $d^{-1}(g(0)-m(0))$ must be one of $\left\{\lambda_{2}, \lambda_{3}, \ldots, \lambda_{n}, \ldots\right\}$, and consequently $k \notin K$. Therefore $k \equiv 0$. (In case (A.2), $d^{-1}(g(0)-m(0))<\lambda_{1}$, so $k \equiv 0$ follows.)

The first equation implies $-\Delta r=M(0,0) r$. Because $M(0,0)>\lambda_{1}$, it follows that $M(0,0) \in\left\{\lambda_{2}, \lambda_{3}, \ldots, \lambda_{n}, \ldots\right\}$ implies $r \notin K$. So $I-L$ is invertible in $W_{y}=\bar{W}_{(0,0)}$ $=K \oplus K$.

Next, we need to verify that $I-A^{\prime}(0,0)=I-L$ is not a surjective map on $\bar{W}_{y}$ when $M(0,0) \in\left\{\lambda_{2}, \lambda_{3}, \ldots, \lambda_{n}, \ldots\right\}$. (Notice that if $\lambda_{1}<M(0,0) \neq \lambda_{i}, i \geqslant 2$, then $I-A^{\prime}(0,0)$ is invertible on $E=C_{0}(\Omega) \oplus C_{0}(\Omega)$. We can thus apply Theorem $\mathrm{D}$ in $\S 2$. We need only to check property $\alpha$ of $A^{\prime}(0,0)$ since $\bar{W}_{(0,0)}=K \oplus K . S_{(0,0)}=0 \oplus 0$, so $A^{\prime}(0,0)$ has property $\alpha$ iff $A^{\prime}(0,0)$ has an eigenvalue $\lambda>1$. Let $\phi_{1}$ be the eigenfunction $-\Delta \phi_{1}=\lambda_{1} \phi_{1} ;$ then $A^{\prime}(0,0)\left(\phi_{1}, 0\right)^{T}=\lambda\left(\phi_{1}, 0\right)^{T}$ where $\lambda=$ $[P+M(0,0)] /\left(P+\lambda_{1}\right)>1$.) Let $M(0,0)=\lambda_{l}$ for some $l \geqslant 2$ and let $\phi_{l}$ be the eigenfunction corresponding to $\lambda_{l}:-\Delta \phi_{l}=\lambda_{l} \phi_{l} . \phi_{l}$ changes sign in $\Omega$. The nodes of $\phi$, divide $\Omega$ into no more than $l$ subdomains. So we can find a function $\phi \in C_{0}(\Omega)$ with $\psi(x)>0$ in $\Omega$, such that $\int_{\Omega} \psi \phi_{l} \neq 0$, say, $\int_{\Omega} \psi \phi_{l}>0$ (the case in which $\int_{\Omega} \psi \phi_{l}<0$ can be treated similarly). Since $\phi_{l}(x)=0$ for $x \in \partial \Omega$, there is a small positive $\delta>0$ along with the $\delta$-neighborhood $U_{\delta}$ of $\partial \Omega$ :

$$
U_{\delta}=[x \in \bar{\Omega} \mid \rho(x, \partial \Omega)<\delta]
$$

such that $\max _{x \in \Omega} \psi(x) \int_{U_{\delta}}\left|\phi_{l}\right|<\frac{1}{5} \int_{\Omega} \psi \phi_{l}$. Denote by $\eta>0$ the number $\min _{x \in \Omega \backslash U_{x}} \psi(x)$. (Recall that $\psi(x)=0$ only if $x \in \partial \Omega$.) See Figure 2 .

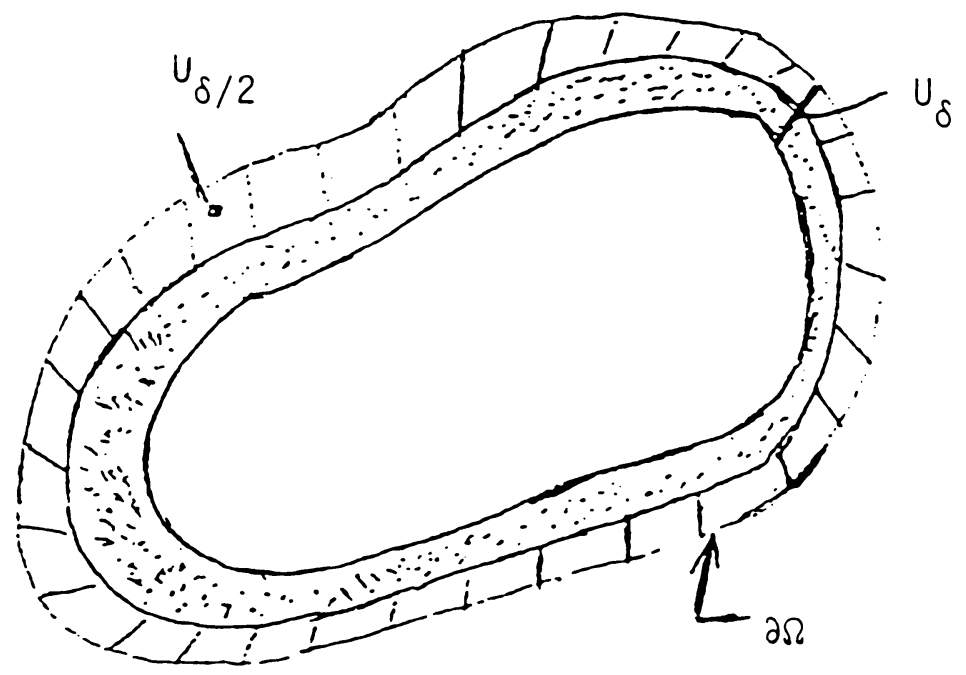

Figure 2 
COEXISTENCE THEOREMS OF STEADY STATES

157

There is a function $\psi_{1}$ in $C_{0}^{\infty}(\Omega)$ which approximates $\psi(x)$ according to the estimate

$$
\max _{x \in \bar{\Omega}}\left|\psi_{1}(x)-\psi(x)\right|<\min \left\{\frac{1}{4}, \frac{1}{4} \eta, \frac{E}{4}\left(\max \left|\phi_{l}(x)\right| \cdot \operatorname{meas} \Omega\right)^{-1}\right\}
$$

where $E=\int_{\Omega} \psi \phi_{l}$. Then $\psi_{1}(x)>0$ for $x \in \bar{\Omega} \backslash U_{\delta}$ and $\int_{\Omega} \psi_{1} \phi_{l}>0$. This is because $\psi_{1}(x)>\psi(x)-\eta / 4>3 \eta / 4>0$ for $x \in \bar{\Omega} \backslash U_{\delta}$ and

$$
\left|\int_{\Omega} \psi_{1} \phi_{l}-\int_{\Omega} \psi \phi_{l}\right| \leqslant \int_{\Omega}\left|\psi_{1}-\psi\right|\left|\phi_{l}\right| \leqslant \frac{E}{4} \text {. }
$$

Therefore

$$
\int_{\Omega} \psi_{1} \phi_{l} \geqslant \int_{\Omega} \phi \phi_{l}-\frac{E}{4}=\frac{3}{4} E>0 .
$$

Let $U_{\delta / 2}=[x \in \bar{\Omega} \mid \rho(x, \partial \Omega)<\delta / 2]$. By a well-known extension theorem in analysis, there is a function $\psi_{2} \in C^{\infty}(\Omega)$ satisfying

$$
\begin{array}{ll}
\psi_{2}(x)=\psi_{1}(x)>0 & \text { for } x \in \bar{\Omega} \backslash U_{\delta}, \\
\psi_{2}(x)=0 & \text { for } x \in \bar{U}_{\delta \backslash 2}, \\
\psi_{2}(x) \geqslant 0 & \text { for } x \in \bar{\Omega} \text { with } \max _{x \in \bar{\Omega}} \psi_{2}(x) \leqslant \max _{x \in \bar{\Omega}} \psi_{1}(x) .
\end{array}
$$

Then

$$
\begin{aligned}
\int_{\bar{\Omega}} \psi_{2} \phi_{l}= & \int_{\bar{\Omega} \backslash U_{\delta}} \psi_{2} \phi_{l}+\int_{U_{\delta}} \psi_{2} \phi_{l}=\int_{\bar{\Omega} \backslash U_{\delta}} \psi_{1} \phi_{l}+\int_{U_{\delta}} \psi_{2} \phi_{l} \\
= & \int_{\bar{\Omega}} \psi_{1} \phi_{l}-\int_{U_{\delta}} \psi_{1} \phi_{l}+\int_{U_{\delta}} \psi_{2} \phi_{l} \\
\geqslant & \int_{\bar{\Omega}} \psi \phi_{l}-\max \left|\psi(x)-\psi_{1}(x)\right| \cdot \int_{\bar{\Omega}}\left|\phi_{l}\right| \\
& -2 \int_{\bar{\Omega}}\left|\phi_{l}\right|\left[\max _{x \in \bar{\Omega}} \psi(x)+\max _{x \in \bar{\Omega}}\left|\psi(x)-\psi_{1}(x)\right|\right] \\
\geqslant & E-\frac{E}{4}-2\left(1+\frac{1}{4}\right) \max _{x \in \bar{\Omega}} \psi(x) \cdot \int_{U_{\delta}}\left|\phi_{l}\right| \\
\geqslant & E-\frac{E}{4}-\frac{10}{4} \times \frac{E}{5}=\frac{E}{4}>0 .
\end{aligned}
$$

So we get a function $0 \leqslant \psi_{2} \in C_{0}^{\infty}(\Omega)$ such that $\int_{\Omega} \psi_{2} \phi_{l} \neq 0$. Now we denote $\phi=(-\Delta+P I) \psi_{2}$; we claim that there is no function $f$ such that $\left(-\Delta-\lambda_{l}\right) f=\phi$ with $\left.f\right|_{\partial \Omega}=0$. If such an $f$ existed, then

$$
\int_{\Omega}\left[\left(-\Delta-\lambda_{l}\right) f\right] \phi_{l}=\int_{\Omega} f\left[\left(-\Delta-\lambda_{l}\right) \phi_{l}\right]=\int_{\Omega} f \cdot 0=0
$$

but the left-hand side equals

$$
\begin{aligned}
\int_{\Omega} \phi \phi_{l} & =\int_{\Omega}\left[(-\Delta+P) \psi_{2}\right] \phi_{l}=\int_{\Omega} \psi_{2}\left[(-\Delta+P) \psi_{2}\right] \phi_{l} \\
& =\int_{\Omega} \psi_{2}\left(\lambda_{l}+P\right) \phi_{l}=\left(\lambda_{l}+P\right) \int_{\Omega} \psi_{2} \phi_{l} \neq 0
\end{aligned}
$$


(We have made use of the zero boundary condition in the above computation.) This contradiction shows the nonexistence of the function $f$.

Now we take

$$
\left(\begin{array}{c}
\psi_{2} \\
\tilde{\psi}
\end{array}\right) \in K \oplus K=\bar{W}_{(0,0)},
$$

where $\tilde{\psi}$ is an arbitrary element in $K$. We claim that there is no function pair $\left(\begin{array}{l}f \\ k\end{array}\right)$ satisfying

$$
\left(\begin{array}{l}
f \\
k
\end{array}\right)-A^{\prime}(0,0)\left(\begin{array}{l}
f \\
k
\end{array}\right)=\left(\begin{array}{c}
\psi_{2} \\
\tilde{\psi}
\end{array}\right)
$$

Suppose there is such an $\left(\begin{array}{l}f \\ k\end{array}\right)$ and recall that

$$
A^{\prime}(0,0)=(-\Delta+P)^{-1}\left[\begin{array}{cc}
M(0,0)+P & 0 \\
0 & P+d^{-1}(g(0)-m(0))
\end{array}\right] .
$$

We have from the first component that

$$
f-(-\Delta+P)^{-1}(M(0,0)+P) f=\psi_{2} .
$$

Since $\psi_{2} \in C_{0}^{\infty}(\Omega)$, by the regularity of $(-\Delta+P)^{-1}, f \in H_{0}^{2}(\Omega)$, and we have $-\Delta f-M(0,0) f=(-\Delta+P) \psi_{2}$, i.e. $\left(-\Delta-\lambda_{l}\right) f=\phi$. (Notice that we have set $\left.M(0,0)=\lambda_{\text {, }}\right)$ This contradicts what we claimed in the preceding paragraph.

So $I-A^{\prime}(0,0)$ is not a surjective map on $\bar{W}_{(0,0)}=K \oplus K$. By Theorem $\mathrm{D}^{\prime}$ in $\S 2$, index $(A,(0,0), K \oplus K)=0$.

(B) We investigate the case of $y=\left(u_{0}, 0\right)$.

In this case $\bar{W}_{y}=C_{0}(\Omega) \oplus K$ and

$$
L=A^{\prime}\left(u_{0}, 0\right)=(-\Delta+P)^{-1}\left[\begin{array}{cc}
f_{u}\left(u_{0}, 0\right)+P & f_{v}\left(u_{0}, 0\right) \\
0 & d^{-1}\left(g\left(u_{0}\right)-m(0)\right)+P
\end{array}\right]
$$

where $f(u, v)=u M(u, v)$. We claim first that $I-L$ is invertible in $\bar{W}_{y}=\bar{W}_{\left(u_{0}, 0\right)}$. Otherwise there is an $\left(\begin{array}{c}r \\ k\end{array}\right) \in \bar{W}_{y}$, such that $\left(\begin{array}{c}r \\ k\end{array}\right)=L\left(\begin{array}{l}r \\ k\end{array}\right)$. This means

$$
\begin{aligned}
-\Delta r=f_{u}\left(u_{0}, 0\right) r+f_{1}\left(u_{0}, 0\right) k, \quad-\Delta k & =d^{-1}\left(g\left(u_{0}\right)-m(0)\right) k, \\
0 & \leqslant k \in K, r \neq \equiv,\left.(r, k)\right|_{\partial \Omega}=(0,0) .
\end{aligned}
$$

The second equation implies $\left[d \Delta+\left(g\left(u_{0}\right)-m(0)\right)\right] k=0$. Suppose $k \not \equiv 0$, then by Lemma 2.1, $\lambda_{1}\left(d \Delta+\left(g\left(u_{0}\right)-m(0)\right) I\right)=0$. This contradicts the assumption that $\lambda_{1}\left(d \Delta+\left(g\left(u_{0}\right)-m(0)\right) I\right)>0$. Therefore $k \equiv 0$. We thus find that the first of the above equations becomes

$$
-\Delta r=f_{u}\left(u_{0}, 0\right) r,\left.\quad r\right|_{a \Omega}=0, \quad r \not \equiv 0 .
$$

This is the linearization of the equation

$$
-\Delta u=f(u, 0)=u M(u, 0),\left.\quad u\right|_{a \Omega}=0
$$

at the point $u_{0}$. Since (4.2) has a unique positive solution $u_{0}$, by Proposition 2.7, equation (4.1) has as its only solution $r \equiv 0$, so we get $k \equiv 0 \equiv r$, provided $\left(\begin{array}{l}r \\ k\end{array}\right)=L\left(\begin{array}{l}r \\ k\end{array}\right)$ for $\left(\begin{array}{l}r \\ k\end{array}\right) \in \bar{W}_{\left(u_{0}, 0\right)} . I-L$ is therefore invertible in $\bar{W}_{\left(u_{0}, 0\right)}$. 
In order to complete the proof of index $\left(A,\left(u_{0}, 0\right), K \oplus K\right)=0$, by Theorem $\mathrm{D}^{\prime}$ in $\S 2$, we claim $I-L=I-A^{\prime}\left(u_{0}, 0\right)$ is either invertible in the whole space $C_{0}(\Omega) \oplus C_{0}(\Omega)$ or is not a surjective map on $\bar{W}_{\left(u_{0}, 0\right)}$. If $I-A^{\prime}\left(u_{0}, 0\right)$ is invertible on $C_{0}(\Omega) \oplus C_{0}(\Omega)$, where

$$
A^{\prime}\left(u_{0}, 0\right)=(-\Delta+P)^{-1}\left[\begin{array}{cc}
f_{u}\left(u_{0}, 0\right)+P & f_{v}\left(u_{0}, 0\right) \\
0 & d^{-1}\left(g\left(u_{0}\right)-m(0)\right)+P
\end{array}\right],
$$

we need to check that $A^{\prime}\left(u_{0}, 0\right)$ has property $\alpha$ on $\bar{W}_{y}=C_{0}(\Omega) \oplus K$, where $y=$ $\left(u_{0}, 0\right), S_{y}=C_{0}(\Omega) \oplus 0$. This means finding a $w \in \bar{W}_{y} \backslash S_{y}$ and a $t \in(0,1)$ such that $w-t A^{\prime} w \in S_{y}$. Since $\lambda_{1}\left[d \Delta+\left(g\left(u_{0}\right)-m(0)\right) I\right]$ by hypothesis is positive, there is a function $\phi>0$ such that

$$
d \Delta \phi+\left(g\left(u_{0}\right)-m(0)\right) \phi=\lambda_{1}\left(d \Delta+\left(g\left(u_{0}\right)-m(0)\right) I\right) \phi>0 \quad \text { in } \Omega .
$$

Therefore,

$$
\left(d^{-1}\left(g\left(u_{0}\right)-m(0)\right)+P\right) \phi>(-\Delta+P) \phi,
$$

i.e.

$$
(-\Delta+P)^{-1}\left(d^{-1}\left(g\left(u_{0}\right)-m(0)\right)+P\right) \phi>\phi .
$$

Denote the operator $(-\Delta+P)^{-1}\left(d^{-1}\left(g\left(u_{0}\right)-m(0)\right)+P\right) I$ by $T$; then $T$ is a compact positive operator and $T \phi>\phi$. By Lemma 2.3, $r(T)>1$. The KreinRutman theorem tells us that there is a function $\psi>0$ in $\Omega$ such that $T \psi=$ $r(T) \psi$. Now $\bar{W}_{y}=C_{0}(\Omega) \oplus K, S_{y}=C_{0}(\Omega) \oplus 0, \bar{W}_{y} \backslash S_{y}=C_{0}(\Omega) \oplus K \backslash\{0\}$. Let $w=\left(\begin{array}{l}0 \\ \psi\end{array}\right) \in \bar{W}_{y} \backslash S_{y}$ and $t=[r(T)]^{-1}$. Then $T \psi=r(T) \psi$ means exactly $w-t A^{\prime} w$ $\in S_{y}$, i.e. $A^{\prime}\left(u_{0}, 0\right)$ has property $\alpha$. So in this case, Theorem D applies; we have thus index $\left(A,\left(u_{0}, 0\right), C_{0}(\Omega) \oplus K\right) \equiv \operatorname{index}_{w}\left(A,\left(u_{0}, 0\right)\right)=0$, since $C_{0}(\Omega) \oplus K \supset K \oplus K$. By the properties of the local fixed point index and the fact that $(K \oplus K)^{0} \neq \varnothing$, we have index $\left(A,\left(u_{0}, 0\right), K \oplus K\right)=0$.

If $I-A^{\prime}\left(u_{0}, 0\right)$ is not invertible in $C_{0}(\Omega) \oplus C_{0}(\Omega)=E$, then there is an $\left(\begin{array}{c}r \\ k\end{array}\right) \in$ $C_{0}(\Omega) \oplus C_{0}(\Omega)$ such that

$$
\left(\begin{array}{l}
r \\
k
\end{array}\right)=A^{\prime}\left(u_{0}, 0\right)\left(\begin{array}{l}
r \\
k
\end{array}\right)
$$

and we get

$$
\left\{\begin{array}{l}
-\Delta r=f_{u}\left(u_{0}, 0\right) r+f_{i}\left(u_{0}, 0\right) k, \\
-\Delta k=d^{-1}\left(g\left(u_{0}\right)-m(0)\right) k,\left.\quad r\right|_{\partial \Omega}=\left.k\right|_{\partial \Omega}=0 .
\end{array}\right.
$$

Case $1 . k \equiv 0$. We get $-\Delta r=f_{u}\left(u_{0}, 0\right) r,\left.r\right|_{\text {a } \Omega}=0$.

By Proposition 2.7 this equation implies $r \equiv 0$. So $r \equiv 0 \equiv k$, and we can rule out this case.

Case 2. $k \not \equiv 0, k \in C_{0}(\Omega)$.

From (4.3) we get $k(x)=(-\Delta+P)^{-1}\left(d^{-1}\left(g\left(u_{0}\right)-m(0)\right)+P\right) k$ so there is a $\psi \in K=\left[\phi \geqslant 0 \mid \phi \in C_{0}(\Omega)\right]$ such that $\int_{\Omega} \psi k \neq 0$. Denote $\phi=(-\Delta+P I)^{-1} \psi$, then $\phi \in K$ by the positivity of the operator $(-\Delta+P)^{-1}$. Consider the element

$$
\left(\begin{array}{c}
\phi_{1} \\
\phi
\end{array}\right) \in W_{\left(u_{0}, 0\right)}=C_{0}(\Omega) \oplus K
$$


where $\phi_{1}$ is an arbitrary element in $C_{0}(\Omega)$. We claim that $\left(\begin{array}{c}\phi_{1} \\ \phi\end{array}\right)$ is not in the range $\left[I-A^{\prime}\left(u_{0}, 0\right)\right] \bar{W}_{y}$. If it is, then there exists a

$$
\left(\begin{array}{l}
r_{1} \\
k_{1}
\end{array}\right) \in C_{0}(\Omega) \oplus K
$$

such that

$$
\left(\begin{array}{l}
r_{1} \\
k_{1}
\end{array}\right)-(-\Delta+P I)^{-1}\left[\begin{array}{cc}
f_{u}\left(u_{0}, 0\right)+P & f_{v}\left(u_{0}, 0\right) \\
0 & d^{-1}\left(g\left(u_{0}\right)-M(0)\right)+P
\end{array}\right]\left(\begin{array}{l}
r_{1} \\
k_{1}
\end{array}\right)=\left(\begin{array}{l}
\phi_{1} \\
\phi
\end{array}\right) .
$$

It follows that $-\Delta k_{1}-d^{-1}\left(g\left(u_{0}\right)-m(0)\right) k_{1}=\psi$. By the definition of $\psi$,

$$
\begin{aligned}
0 & \neq \int_{\Omega} \psi k=\int_{\Omega}\left(-\Delta k_{1}-d^{-1}\left(g\left(u_{0}\right)-m(0)\right) k_{1}\right) k \\
& =\int_{\Omega} k_{1}\left[-\Delta-d^{-1}\left(g\left(u_{0}\right)-m(0)\right)\right] k=0,
\end{aligned}
$$

by (4.3). This contradiction establishes our claim. So $I-A^{\prime}\left(u_{0}, 0\right)$ is not a surjective map on $\bar{W}_{\left(u_{0}, 0\right)}$. Therefore Theorem $\mathrm{D}^{\prime}$ applies, and we come to the conclusion that index $\left(A,\left(u_{0}, 0\right), C_{0}(\Omega) \oplus K\right)=0$ and therefore index $\left(A,\left(u_{0}, 0\right), K+K\right)=0$.

(C) We investigate the case of $y=\left(0, v_{0}\right)$.

(Note: in case of (A.2), our proof is completed, since $v_{0}=0$.) The discussion for this case is quite similar to that of (B); we just simply give the outline of the proof. In this case

$$
\begin{aligned}
L & =A^{\prime}\left(0, v_{0}\right) \\
& =(-\Delta+P)^{-1}\left[\begin{array}{cc}
M\left(0, v_{0}\right)+P & 0 \\
d^{-1} v_{0} g^{\prime}(0) & d^{-1}\left(g(0)-m\left(v_{0}\right)-v_{0} m^{\prime}\left(v_{0}\right)\right) I+P
\end{array}\right] .
\end{aligned}
$$

(i) We show $I-L$ is invertible on $\bar{W}_{\left(0, v_{0}\right)}=K \oplus C_{0}(\bar{\Omega}) . L\left(\begin{array}{c}r \\ k\end{array}\right)=\left(\begin{array}{c}r \\ k\end{array}\right)$ implies

$$
\begin{aligned}
& -\Delta r=M\left(0, v_{0}\right) r, \\
& -\Delta k=d^{-1} v_{0} g^{\prime}(0) r+d^{-1}\left(g(0)-m\left(v_{0}\right)-v_{0} m^{\prime}\left(v_{0}\right)\right) k, \\
& \left.r\right|_{\partial \Omega}=\left.k\right|_{\partial \Omega}=0 .
\end{aligned}
$$

Suppose $0 \not \equiv r \in K$. From the first equation and Lemma 2.1 it follows that $\lambda_{1}\left(\Delta+M\left(0, v_{0}\right)\right)=0$, which contradicts our assumption that $\lambda_{1}\left(\Delta+M\left(0, v_{0}\right)\right)>0$. So $r \equiv 0$. Consequently by Proposition 2.7 we have $k \equiv 0$.

(ii) We show $I-L$ is either invertible in the whole space $C_{0}(\Omega) \oplus C_{0}(\Omega)=E$ or not a surjective map on $\bar{W}_{\left(0, v_{0}\right)}$.

If $I-A^{\prime}\left(0, v_{0}\right)$ is not invertible on $E$, there exists $\left(\begin{array}{l}r \\ k\end{array}\right)$ with

$$
\left(\begin{array}{l}
r \\
k
\end{array}\right)=A^{\prime}\left(0, v_{0}\right)\left(\begin{array}{l}
r \\
k
\end{array}\right) \quad \text { where } 0 \not \equiv\left(\begin{array}{l}
r \\
k
\end{array}\right) \in C_{0}(\Omega) \oplus C_{0}(\Omega) .
$$


This means

$$
\begin{aligned}
& -\Delta r=M\left(0, v_{0}\right) r, \\
& -d \Delta k=v_{0} g^{\prime}(0) r+\left(g(0)-m\left(v_{0}\right)-v_{0} m^{\prime}\left(v_{0}\right)\right) k, \\
& \left.r\right|_{\partial \Omega}=\left.k\right|_{\partial \Omega}=0 .
\end{aligned}
$$

If $r \equiv 0$, then we have $k \equiv 0$ follows from Proposition 2.7.

If $r \not \equiv 0$, remember we have shown in (i) that $I-L$ is invertible on $\bar{W}_{y}=\bar{W}_{\left(0, v_{0}\right)}$. So by Theorem $\mathrm{D}^{\prime}$ we need only verify that $I-L$ is not a surjective map. Since $r \not \equiv 0$, there is a $\psi \in K$, such that $\int_{\Omega} \psi r \neq 0$. Denote $\phi=(-\Delta+P)^{-1} \psi \in K$. Consider $\left(\begin{array}{c}\phi \\ \phi_{1}\end{array}\right) \in \bar{W}_{\left(0, v_{0}\right)}=K \oplus C_{0}(\Omega)$ where $\phi_{1}$ is an arbitrary element in $C_{0}(\Omega)$. We claim that $\left(\begin{array}{c}\phi \\ \phi_{1}\end{array}\right)$ is not in the range $(I-L) \bar{W}_{\left(0, v_{0}\right)}$. If it were, then there exists

$$
\left(\begin{array}{l}
r_{1} \\
k_{1}
\end{array}\right) \in \bar{W}_{\left(0, v_{0}\right)}=K \oplus C_{0}(\Omega)
$$

such that

$$
\begin{aligned}
\left(\begin{array}{l}
r_{1} \\
k_{1}
\end{array}\right) & -(-\Delta+P)^{-1}\left[\begin{array}{cc}
M\left(0, v_{0}\right)+P & 0 \\
d^{-1} v_{0} g^{\prime}(0) & d^{-1}\left(g(0)-m\left(v_{0}\right)-v_{0} m^{\prime}\left(v_{0}\right)\right)+P
\end{array}\right]\left(\begin{array}{l}
r_{1} \\
k_{1}
\end{array}\right) \\
= & \left(\begin{array}{c}
\phi \\
\phi_{1}
\end{array}\right) .
\end{aligned}
$$

This implies $(-\Delta+P) r_{1}-\left(M\left(0, v_{0}\right)+P\right) r_{1}=(-\Delta+P) \phi$; we thus have

$$
-\Delta r_{1}-M\left(0, v_{0}\right) r_{1}=\psi
$$

and

$$
0 \neq \int_{\Omega} \psi r=\int_{\Omega}\left(-\Delta r_{1}-M\left(0, v_{0}\right) r_{1}\right) r=\int_{\Omega} r_{1}\left(-\Delta r-M\left(0, v_{0}\right) r\right)=0
$$

because $-\Delta r=M\left(0, v_{0}\right) r$. This contradiction shows $I-A^{\prime}\left(0, v_{0}\right)$ is not a surjection. Consequently Theorem $\mathrm{D}^{\prime}$ applies and index $\left(A,\left(0, v_{0}\right), K \oplus K\right)=0$.

(iii) Finally, we must show that if $I-A^{\prime}\left(0, v_{0}\right)$ is invertible on $E$, then $L$ has property $\alpha$. Since $S_{\left(0, v_{0}\right)}=0 \oplus C_{0}(\bar{\Omega})$, we have to find a $w \in \bar{W}_{y} \backslash S_{y}$ with $w-t A^{\prime} w$ $\in S_{y}$, where $t \in(0,1)$. Since $\mu_{1}=\lambda_{1}\left(\Delta+M\left(0, v_{0}\right) I\right)>0$, there is a positive eigenfunction $\phi$ such that $\left(\Delta+M\left(0, v_{0}\right)\right) \phi=\mu_{1} \phi>0$. This implies $(-\Delta+P) \phi<$ $\left(M\left(0, v_{0}\right)+P\right) \phi$. Then by Lemma 2.3, $r(T)=r\left[(-\Delta+P)^{-1}\left(M\left(0, v_{0}\right)+P\right)\right]>1$ where $T$ denotes the operator $(-\Delta+P)^{-1}\left(M\left(0, v_{0}\right)+P\right)$ which is compact and positive. According to the Krein-Rutman theorem, there exists a $\psi \in K$ such that

$$
(-\Delta+P)^{-1}\left(M\left(0, v_{0}\right)+P\right) \psi=r(T) \psi .
$$

Now let $w=\left(\begin{array}{l}\psi \\ 0\end{array}\right) \in \bar{W}_{y} \backslash S_{y}=K \oplus C_{0}(\bar{\Omega})$, where $y=\left(0, v_{0}\right)$. Then it is easy to check $w-(1 / r(T)) A^{\prime} w \in S_{\left(0, v_{0}\right)}=0 \oplus C_{0}(\bar{\Omega})$. Notice that $t=1 / r(T)<1$. This concludes the proof of property $\alpha$. Therefore Theorem D applies, and

$$
\text { index }\left(A,\left(0, v_{0}\right), K \oplus K\right)=0 \text {. }
$$


Combining these facts we have proved that

$$
\begin{aligned}
0 & =i n d e x(A,(0,0), K \oplus K)=\operatorname{index}\left(A,\left(u_{0}, 0\right), K \oplus K\right) \\
& =i n d e x\left(A,\left(0, v_{0}\right), K \oplus K\right)
\end{aligned}
$$

and $\operatorname{deg}(A$, int $D, K \oplus K)=1$. We hence have proved the existence of a positive solution of (1).

REMARK. We have gone through the proof for Theorem 1.A. We have to point out here the places where extra arguments are needed when going through the proof of Theorem 1.B.

(i) Instead of the parametrized system (1.A) $)_{\theta}$ we use the system

$$
\left\{\begin{array}{l}
-\Delta u=\theta u M(u, v), \\
-d \Delta v=v g(u),\left.\quad(u, v)\right|_{\partial \Omega}=(0,0) .
\end{array}\right.
$$

From Theorem $\mathrm{A}^{\prime}$ in $\S 2$, the upper bound of $v$ is independent of the diffusion rate $d_{0}=\theta^{-1}$, so we can assume that the positive solution $\left(u_{\theta}, v_{\theta}\right)$ of $(1 . \mathrm{B})_{\theta}$ satisfies $v_{\theta} \leqslant B_{2}$ for all $\theta \in(0, \infty)$. Of course $0 \leqslant u_{\theta} \leqslant B_{1}=C_{0}+\varepsilon$ still holds (see $\S 3$ ).

(ii) The other arguments of the proof of Theorem (1.B) are even simpler than those in the proof of Theorem 1.A, because in this csase $m \equiv 0$.

Corollary. Assume $m(0)-g(0) \geqslant 0$. Then the system (1) has a positive solution iff

$$
r\left[\left(-\Delta+\frac{m(0)-g(0)}{d}\right)^{-1}\left(\frac{g\left(u_{0}\right)-g(0)}{d}\right) I\right]>1,
$$

where $r(L)$ represents the spectral radius of $L$.

Proof. Notice first that $d^{-1}(m(0)-g(0)) \geqslant 0$. So $-\Delta+d^{-1}(m(0)-g(0)) I$ is invertible and the operator in brackets is compact and positive. We first show the necessity. (1) has a positive solution implies $\lambda_{1}\left(d \Delta+\left(g\left(u_{0}\right)-m(0)\right) I\right)>0$ by Theorem 1.A, so there is a positive eigenfunction $\psi>0$ :

$$
d \Delta \psi+\left(g\left(u_{0}\right)-m(0)\right) \psi=\nu_{1} \psi>0,
$$

where $\nu_{1}=\lambda_{1}\left(d \Delta+\left(g\left(u_{0}\right)-m(0)\right) I\right)$ and $\left.\psi\right|_{\partial \Omega}=0$. Therefore, we have

$$
d^{-1}\left(g\left(u_{0}\right)-g(0)\right) \psi>\left(-\Delta+(m(0)-g(0)) d^{-1}\right) \psi .
$$

So

$$
\left(-\Delta+\frac{m(0)-g(0)}{d}\right)^{-1}\left(\frac{g\left(u_{0}\right)-g(0)}{d}\right) \psi>\psi,
$$

and the result we want to prove follows from Lemma 2.3 .

We next show the sufficiency. Since the operator in the brackets is compact and positive, by the Krein-Rutman theorem, there is a positive function $\psi$ on $\Omega$ such that

$$
\left(-\Delta+\frac{m(0)-g(0)}{d}\right)^{1}\left(\frac{g\left(u_{0}\right)-g(0)}{d}\right) \psi=r \psi
$$


where $r>1$ is the spectral radius. A simple computation shows

$$
-d \Delta \psi=\left(\left(\frac{g\left(u_{0}\right)-g(0)}{r}\right)-(m(0)-g(0))\right) \psi .
$$

Since $\psi>0, g\left(u_{0}\right)-g(0) \geqslant 0$ and $r>1$, it is obvious that

$$
-d \Delta \psi \leqslant\left[g\left(u_{0}\right)-g(0)-(m(0)-g(0))\right] \psi=\left(g\left(u_{0}\right)-m(0)\right) \psi .
$$

Integrating by parts over $\Omega$ yields

$$
d \int_{\Omega}|\nabla \psi|^{2}<\int_{\Omega}\left(g\left(u_{0}\right)-m(0)\right) \psi^{2} .
$$

Thus

$$
-d \int_{\Omega}|\nabla \psi|^{2}+\int_{\Omega}\left(g\left(u_{0}\right)-m(0)\right) \psi^{2}>0
$$

Since

$$
\nu_{1}=\sup _{\phi \in H_{0}^{1}(\Omega)} \frac{-d \int_{\Omega}|\nabla \phi|^{2}+\int_{\Omega}\left(g\left(u_{0}\right)-m(0)\right) \phi^{2}}{\int_{\Omega} \phi^{2}},
$$

hence $\nu_{1}>0$, i.e. $\lambda_{1}\left(d \Delta+\left(g\left(u_{0}\right)-m(0)\right) I\right)=\nu_{1}>0$. Now the desired conclusion follows immediately from Theorem 1.A. The argument to prove the assertion for (1.B) is quite analogous to that given above.

Note. This corollary generalizes the main result of $\$ 1$ in [12].

Before going to our next theorem, a lemma is in order which generalizes the one given by Conway, Gardner and Smoller [8] in the one dimensional setting in a special form (see Theorem 2.7 in their joint paper).

Lemma 4.1. Let $A=\sum_{i j} a_{i j}(x) D_{i} D_{j}$ where $a_{i j}=a_{j i}$. Let $B=\sum_{i j} b_{i j}(x) D_{i} D_{j}$, $b_{i j}=b_{j i}$. Assume $A$ and $B$ are uniformly elliptic operators with zero Dirichlet boundary conditions on $\Omega \subset \mathbf{R}^{n}$. Let

$$
L:\left(\begin{array}{c}
w \\
z
\end{array}\right) \mapsto\left(\begin{array}{c}
A w+a(x) w+b(x) z \\
B z+c(x) z
\end{array}\right)
$$

map $C_{0}^{2+\alpha}(\Omega)$ to $C_{0}^{\alpha}(\Omega)$. Then the spectrum of $L$ consists of only the pure point spectrum

$$
\sigma_{p}=\left\{\xi_{1}, \xi_{2}, \ldots, \xi_{n}, \ldots\right\} \cup\left\{\theta_{1}, \theta_{2}, \ldots, \theta_{n}, \ldots\right\},
$$

where we denote by $\xi_{1}>\xi_{2}>\cdots>\xi_{n}>\cdots$ the pure point spectrum of the operator $B+c(x) I$, while $\theta_{1}>\theta_{2}>\cdots>\theta_{n}>\cdots$ is the pure point spectrum of the operator $A+a(x) I$.

The proof of Lemma 4.1 is a quite straightforward analogue of the original given in Conway, Gardner and Smoller [8].

We assume hereafter $M(0,0) \neq \lambda_{1}, g(0)-m(0) \neq \lambda_{1} d$, and in case that $m \equiv 0$, we make all the assumptions in Theorem 1.B. Then we have

THEOREM 4.2. The system (1) has a positive solution iff all its trivial nonnegative solutions are unstable. 
Proof. (I) The necessity part. As we have seen, the possible trivial nonnegative solutions are $(0,0),\left(u_{0}, 0\right),\left(0, v_{0}\right)$. To prove the theorem we need only investigate the linearization of the operator $F$ at these trivial solutions, where $F$ is

$$
F=\left(\begin{array}{l}
u \\
v
\end{array}\right) \mapsto\left(\begin{array}{c}
\Delta u+u M(u, v) \\
d \Delta v+v(g(u)-m(v))
\end{array}\right) .
$$

First of all, let $(u, v)$ be a positive solution of (1). Then $u_{0}>u>0$, so by Lemma $1.1 M(0,0)>\lambda_{1}$. We have

$$
F_{u, v}(0,0)=\left[\begin{array}{cc}
\Delta+M(0,0) I & 0 \\
0 & d \Delta+(g(0)-m(0)) I
\end{array}\right] .
$$

Since $M(0,0)>\lambda_{1}$, therefore $\lambda_{1}(\Delta+M(0,0) I)>0$ and the spectrum of $F_{u, v}(0,0)$ contains a positive point so $(0,0)$ is unstable. Next at $\left(u_{0}, 0\right)$, we have

$$
\begin{gathered}
F_{u, v}\left(u_{0}, 0\right)=\left[\begin{array}{cc}
\Delta+M\left(u_{0}, 0\right)+u_{0} M_{u}\left(u_{0}, 0\right) I & u_{0} M_{v}\left(u_{0}, 0\right) I \\
0 & d \Delta+\left(g\left(u_{0}\right)-m(0)\right) I
\end{array}\right], \\
F_{u, v}\left(u_{0}, 0\right)\left(\begin{array}{c}
w \\
z
\end{array}\right)=\left[\begin{array}{c}
\Delta w+\left[M\left(u_{0}, 0\right)+u_{0} M_{u}\left(u_{0}, 0\right)\right] w+u_{0} M_{v}\left(u_{0}, 0\right) z \\
d \Delta z+\left(g\left(u_{0}\right)-m(0)\right) z
\end{array}\right] .
\end{gathered}
$$

By Lemma 4.1, $F_{u, v}\left(u_{0}, 0\right)$ has only pure point spectrum $\sigma_{p}$ given by $\sigma_{p}=$ $\left\{\xi_{1}, \xi_{2}, \ldots\right\} \cup\left\{\theta_{1}, \theta_{2}, \ldots\right\}$ where $\left\{\xi_{1}, \xi_{2}, \ldots\right\}$ is the point spectrum of the operator $\Delta+\left[M\left(u_{0}, 0\right)+u_{0} M_{u}\left(u_{0}, 0\right)\right] I$ while $\left\{\theta_{1}, \theta_{2}, \ldots\right\}$ is the point spectrum of the operator $d \Delta+\left(g\left(u_{0}\right)-m(0)\right) I$. From Theorem 1.A,

$$
\theta_{1}=\lambda_{1}\left(d \Delta+\left(g\left(u_{0}\right)-m(0)\right) I\right)>0 .
$$

This means $\left(u_{0}, 0\right)$ is an unstable solution because $\sigma_{p}$ contains a positive member.

In case that system $(1)$ has the third trivial solution $\left(0, v_{0}\right)$ (not always!), then by Lemma 1.1, $g(0)>\lambda_{1} d+m(0)$. We have

$$
F_{u, v}\left(0, v_{0}\right)=\left[\begin{array}{cc}
\Delta+M\left(0, v_{0}\right) I & 0 \\
v_{0} g^{\prime}(0) & d \Delta+\left(g(0)-m\left(v_{0}\right)-v_{0} m^{\prime}\left(v_{0}\right)\right) I
\end{array}\right]
$$

and

$$
F_{u, v}\left(0, v_{0}\right)\left(\begin{array}{c}
w \\
z
\end{array}\right)=\left[\begin{array}{c}
\Delta w+M\left(0, v_{0}\right) w \\
v_{0} g^{\prime}(0) w+d \Delta z+\left(g(0)-m\left(v_{0}\right)-v_{0} m^{\prime}\left(v_{0}\right)\right) z
\end{array}\right] .
$$

By Theorem 1.A again, $\lambda_{1}\left(\Delta+M\left(0, v_{0}\right) I\right)>0$. Using Lemma 4.1 (strictly speaking, an analogue of Lemma 4.1), we see that the spectrum of $F_{u, v}\left(0, v_{0}\right)$ contains a positive number. Therefore $\left(0, v_{0}\right)$ is unstable too.

(II) The sufficiency part. First of all, $(0,0)$ is a trivial nonnegative solution with

$$
F_{u, v}(0,0)=\left[\begin{array}{cc}
\Delta+M(0,0) I & 0 \\
0 & d \Delta+(g(0)-m(0)) I
\end{array}\right] .
$$

So either $\lambda_{1}(\Delta+M(0,0) I)>0$ or $\lambda_{1}(d \Delta+(g(0)-m(0)) I)>0$, i.e., either $M(0,0)>\lambda_{1}$ or $g(0)>\lambda_{1} d+m(0)$. The possible cases are

(i) $M(0,0)>\lambda_{1}, g(0)<\lambda_{1} d+m(0)$,

(ii) $M(0,0)>\lambda_{1}, g(0)>\lambda_{1} d+m(0)$,

(iii) $M(0,0)<\lambda_{1}, g(0)>\lambda_{1} d+m(0)$. 
We go through case (i) first. Since $M(0,0)>\lambda_{1}$, by Lemma 1.1 , we have a trivial nonnegative solution $\left(u_{0}, 0\right)$. By our assumption it is unstable. The spectrum $\sigma_{p}$ of $F_{u, v}\left(u_{0}, 0\right)$ contains a positive eigenvalue; according to Lemma $4.1 \sigma_{p}=\left\{\xi_{1}, \xi_{2}, \ldots\right\}$ $\cup\left\{\theta_{1}, \theta_{2}, \ldots\right\}$ where $\xi_{1}>\xi_{2}>\cdots$ comprise the point spectrum of the operator $\Delta+\left[M\left(u_{0}, 0\right)+u_{0} M_{u}\left(u_{0}, 0\right)\right] I$ which is the linearization of the operator $u \mapsto \Delta u+$ $u M(u, 0)$ at $u_{0}$. It follows from Proposition 2.7 that $\xi_{1}<0$. This means that $\left\{\theta_{1}, \theta_{2}, \ldots\right\}$ contains a positive number, because $\theta_{1}>\theta_{2}>\cdots$, therefore, $\theta_{1}>0$. $\left(\left\{\theta_{1}, \theta_{2}, \ldots\right\}\right.$ is the point spectrum of the operator $d \Delta+\left(g\left(u_{0}\right)-m(0)\right) I$. $)$ It follows from Theorem 1.A or 1.B that system (1) has a positive solution.

An argument similar to the above will work equally well for case (ii). Finally, we point out that case (iii) will not occur. Since $g(0)>\lambda_{1} d+m(0)$ by Lemma 1.1 , the equation

$$
-d \Delta v=v(g(0)-m(v)),\left.\quad v\right|_{\partial \Omega}=0,
$$

has a unique positive solution $v_{0}$, i.e., the system (1) has a trivial nonnegative solution $\left(0, v_{0}\right)$. By our assumption it is supposed to be unstable. So $F_{u, v}\left(0, v_{0}\right)$ by definition, has a positive number in its point spectrum $\sigma_{p}$. According to Lemma 4.1, $\sigma_{p}$ consists of $\left\{\xi_{1}, \xi_{2}, \ldots\right\} \cup\left\{\theta_{1}, \theta_{2}, \ldots\right\}$ where $\xi_{1}>\xi_{2}>\cdots$ are eigenvalues of the operator $\Delta+M\left(0, v_{0}\right) I$ and $\theta_{1}>\theta_{2}>\cdots$ are eigenvalues of the operator $d \Delta+\left(g(0)-m\left(v_{0}\right)-v_{0} m^{\prime}\left(v_{0}\right)\right) I$ (see part (I) of our proof). By Proposition 2.7, $\theta_{1}<0$, so $\xi_{1}$ must be positive, i.e., $\lambda_{1}\left(\Delta+M\left(0, v_{0}\right) I\right)>0$. But $M(0,0) \geqslant M\left(0, v_{0}\right)$ by hypothesis $\left(\mathrm{M}_{1}\right)$, so we have $\lambda_{1}(\Delta+M(0,0) I)>0$, which implies $M(0,0)>\lambda_{1}$. Therefore case (iii) is impossible. This completes our proof.

ACKNOWLEDGMENTS. This work is part of the author's Ph.D. dissertation at Tulane University, 1986. The author wishes to express his sincere appreciation to the late Professor Edward D. Conway for his guidance. The author wishes also to express his gratitude to Professors J. A. Goldstein and Joel Smoller for their many very helpful and important suggestions.

\section{BIBLIOGRAPHY}

1. N. Alikakos, I." bounds of solutions of reaction-diffusion equations, Comm. Partial Differential Equations 4 (1979), 827-868.

2. H. Amann, Fixed point equations and nonlinear eigenwalue problems in ordered Banach spaces, SIAM Rev. 18 (1976), 620-709.

3. H. Berestyski, Le nombre de solutions de certains problemes semi-lineaires elliptiques, J. Funct. Anal. 40 (1981), 1-29.

4. H. Berestyski and P. L. Lions, Some applications of the method of super and subsolutions, Lecture Notes in Math., vol. 782, Springer-Verlag, Berlin, 1980, pp. 16-42.

5. J. Blat and K. J. Brown, Bifurcation of steady-state solution in predator-prey and competition sistems, Proc. Roy. Soc. Edinburgh 97A (1984), 21-34.

6. E. D. Conway, Diffusion and the predator-prey interaction: patterns in closed sistems, Partial Differential Equations and Dynamical Systems (W. E. Fitzgibon, III, ed.), Pitman, London, 1984. pp. $85-133$.

7. Diffusion and the predator-prey interaction: steady states with flux at the boundary, Contemporary Math., vol. 17, Amer. Math. Soc., Providence, R. I., 1983, pp. 215-234.

8. E. D. Conway, R. Gardner and J. Smoller, Stability and bifurcation of steady state solutions for predator-prel equations, Adv. Appl. Math. 3 (1982), 288-334.

9. E. D. Conway and J. Smoller, Diffusion and the predator-prey interaction, SIAM J. Appl. Math. 33 (1977), 673-686. 
10. M. G. Crandall and P. H. Rabinowitz, Bifurcation, perturbation of simple eigenvalues and linearized stability, Arch. Rational Mech. Anal. 52 (1973), 161-181.

11. E. N. Dancer, On the indices of fixed points of mappings in cones and applications, J. Math. Anal. Appl. 91 (1983), 131-151.

12. $\ldots$, On positive solutions of some partial differential equations, Trans. Amer. Math. Soc. 284 (1984), 729-743.

13. J. A. Goldstein, Semigroups of linear operators and applications, Oxford Univ. Press, New York, 1985.

14. P. Korman and A. Leung, A general monotone scheme for elliptic system with applications to ecological models, Proc. Roy. Soc. Edinburgh 102A (1986), 315-325.

15. A. Leung, Monotone schemes for semilinear elliptic systems realted to ecology, Math. Meth. Appl. Sci. 4 (1982), 272-285.

16. P. De Mottoni and F. Rothe, Convergence to homogeneous equilibrium states for generalized Volterra-Lotka systems, SIAM J. Appl. Math. 37 (1979), 648-663.

17. C. V. Pao, On nonlinear reaction-diffusion systems, J. Math. Anal. Appl. 87 (1982), 165-198.

18. J. Smoller, Shock waves and reaction-diffusion equations, Springer-Verlag, New York, 1983.

19. J. Smoller, A. Tromba and A. Wasserman, Nondegenerate solutions of boundary-value problems, Nonlinear Anal. 4 (1980), 207-215.

Department of Mathematics, Tulane University, New Orleans, Louisiana 70118

Department of Mathematics, Kansas State University, Manhattan, Kansas 66506 (Current address) 